MPS-RR 2000-43

math-ph/0011002

\title{
Unitary Irreducible Representations of a Lie Algebra for Matrix Chain Models
}

\author{
H. P. Jakobsen ${ }^{a \text {, }}$ and C.-W. H. Lee , $^{a, b}$, 色 \\ ${ }^{a}$ Department of Mathematics, University of Copenhagen, \\ Universitetsparken 5, DK-2100 Copenhagen, Denmark. \\ ${ }^{b}$ MaPhySto - Centre of Mathematical Physics and Stochastics
}

1 November, 2000

\begin{abstract}
There is a decomposition of a Lie algebra for open matrix chains akin to the triangular decomposition. We use this decomposition to construct unitary irreducible representations. All multiple meson states can be retrieved this way. Moreover, they are the only states with a finite number of nonzero quantum numbers with respect to a certain set of maximally commuting linearly independent quantum observables. Any other state is a tensor product of a multiple meson state and a state coming from a representation of a quotient algebra that extends and generalizes the Virasoro algebra. We expect the representation theory of this quotient algebra to describe physical systems at the thermodynamic limit.
\end{abstract}

AMS classification numbers: 17B65, 17B35, 17B10, 81V22, 81V05.

PACS numbers: 02.10.Tq, 11.15.Pg, 12.15.Sq.

Keywords: lowest weight representation, triangular decomposition, large- $N$ limit, mesons, string bit model.

\footnotetext{
${ }^{1}$ e-mail address: jakobsen@math.ku.dk

${ }^{2}$ e-mail address: lee@math.ku.dk

${ }^{3}$ funded by a grant from the Danish National Research Foundation.
} 


\section{Introduction}

Theories with matrix degrees of freedom are of wide interest in physics. Quantum chromodynamics (QCD) is an important example. Each gluon field carries two color indices. They can be treated as row and column indices of a matrix field. A typical term in the action of a physical theory is constructed by multiplying matrix fields together and taking the trace of the resulting product; this serves to preserve gauge invariance. M(atrix)-Theory [1], a candidate for a unified theory of gravitational, strong and electroweak interactions, is another major example. In this model, the matrices describe the positions of D0-branes and their relative distances noncommutatively [2].

So far, the most successful calculational tool for both theories is perturbative analysis, whose approximation assumptions are valid in the high-energy regime of QCD and the classical limit of M-theory. Indeed, there is an excellent agreement between perturbative QCD predictions and measurements of high-energy scattering experiments among quarks and gluons. (See Ref.[3], for instance, for a general introduction and further literature on the subject.) Perturbative M-theory calculations of scattering processes among M-theory objects are, by and large, in good agreements with classical supergravity, too. (Ref. [4] lists two latest reviews on the subject. Further literature can be found therein.) To study important low-energy phenomena of QCD like color confinement, hadron spectrum or the parton distribution of a nucleon, or large quantum effects of supergravity, however, it is necessary to develop non-perturbative methods.

As we have just noted, both QCD and M-theory are intrinsically matrix models. Little is known about the ramifications of the matrix nature, though many researchers believe that this is the key to a deeper understanding of the physics of a matrix model. One approach is to study its symmetry. This consists in identifying a symmetry of a generic matrix model, expressing the symmetry in terms of a Lie algebra (or quantum group) and developing a representation theory for the Lie algebra.

Numerous examples have demonstrated the fruitfulness of studying representation theories. To name but a few, the representation theory of so(3) shapes the energy spectra of physical systems with rotational symmetry; the representation theory of the Poincaré algebra enables us to classify massless fundamental particles [3]; even more remarkably, the so(4) symmetry of the hydrogenic atom dictates its energy spectrum completely [0].

Perhaps the most prominent example in recent years is the Virasoro al- 
gebra, a Lie algebra describing two-dimensional conformal symmetry. Its representation theory reveals how the reducibility of a highest weight representation depends on the values of $c$, the central charge, and $h$, the eigenvalue of the highest weight state under the action of $L_{0}$, the energy operator. We can use these irreducible representations to describe compactified string theory [6]. We can also use a small number of highest weight reducible representations to build up a so-called minimal model describing a physical system at criticality like the Ising model and the three-state Potts model [7]. In addition, the representation theory renders us a character formula

$$
\operatorname{Tr} \exp \left[2 \pi \mathrm{i} \tau\left(L_{0}-\frac{c}{24}\right)\right]=\frac{q^{h+(1-c) / 24}}{\eta(\tau)},
$$

where $\tau$ is a complex variable, Tr means a sum over all states of highest weight representation and $\eta(\tau)$ is the Dedekind function

$$
\eta(\tau)=\exp \left(\frac{\pi \mathrm{i} \tau}{12}\right) \prod_{n=1}^{\infty}(1-\exp (2 \pi \mathrm{i} n \tau)) .
$$

If we interpret $\tau$ as the ratio between two complex periods along two linearly independent directions on a torus, this character formula becomes nothing but the holomorphic part of the partition function of a conformal field theory on a torus [8]. Thus we can solve for the thermodynamics of this system.

In Ref. [9], Rajeev and one of us gave an exposition on the basic properties of a newly discovered Lie algebra $\hat{G}_{\Lambda, \Lambda_{F}}$ for open matrix chains in the large- $N$ limit [10]. (By an open matrix chain we mean a state produced by the action of a product of a row vector, several square matrices and a column vector of creation operators on the vacuum.) They can be interpreted as mesons in QCD, discretized open strings in a string-bit model [11] or one-dimensional open quantum spin chain systems. The relation of this Lie algebra with another Lie algebra for closed matrix chains was discussed at length in Ref. 12]. We would like to build upon the results of Ref. [9], and work out a representation theory for it. In this article, we will present first results on the subject.

As noted in Ref. [12], $\hat{G}_{\Lambda, \Lambda_{F}}$ can be broken down into a direct sum of subalgebras in a manner similar to the triangular decomposition of a semisimple Lie algebra. Just as a traditional triangular decomposition gives rise to lowest weight representations, this decomposition for $\hat{G}_{\Lambda, \Lambda_{F}}$ leads to interesting representations generated by a weight vector, which we will call 
a lowest weight vector. The corresponding representation will be called a lowest weight representation. It can be made irreducible by quotienting out the maximal subrepresentation. Some lowest weight vectors produce unitary representations.

Since the Cartan subalgebra we have found for $\hat{G}_{\Lambda, \Lambda_{F}}$ is simultaneously a maximally commutative subalgebra, we can treat it as a linear space generated by a maximally commuting set of linearly independent quantum observables. A lowest weight vector is then a quantum eigenstate of this set of quantum observables, and the lowest weight a set of quantum numbers. An interesting result we are going to show is that if only a finite number of these quantum numbers are non-zero, then this eigenstate must be, in the context of QCD, a multiple meson state. Any state with an infinite number of non-zero quantum numbers must be a tensor product of a multiple meson and a state coming from an irredicible representation of a certain quotient algebra which extends and generalizes the Virasoro algebra. Already for the case $\Lambda=1$ the quotient algebra is quite interesting. Specifically, it is an extension of the Virasoro algebra by an infinite Heisenberg algebra [13]. We expect the representation theory of the quotient algebra to describe physical systems at the thermodynamic limit.

This paper is organized as follows. We will review without proofs the definition of $\hat{G}_{\Lambda, \Lambda_{F}}$ and its basis properties in Section 2, further details of which can be found in Refs. [9] and [12]. We will work out two useful bases for the Lie algebra in Section 3, and its Cartan subalgebra and root vecotors in Section 4 . (The reader is advised to read only the statements of the propositions in these two sections on a first reading, and return to them later on if he or she is interested in the details.) We will define the notion of a Verma-like module and the associated Hermitian form in Section 5, and use this to identify the representation spaces of multiple meson states in Section 6 and other states which are related to the quotient algebra in Section 7 .

We follow Refs. [14] and [15] in the usage of Lie algebra terminologies.

\section{Definitions}

Two Lie algebras were defined in Ref. [12]: the grand string algebra and the open string algebra. The latter is our major interest in this article, and was defined as a quotient of the former. We will briefly review them in this section. Further details of the notations and formalism can be found in 
Refs. [9] and [12]. One agreement we need to make with the reader now is that unless otherwise specified, the summation convention will not be adopted.

\begin{tabular}{||c|c||}
\hline $\begin{array}{c}\text { operator of } \\
\text { which kind }\end{array}$ & expression \\
\hline \hline first & $\Xi_{\lambda_{2}}^{\lambda_{1}} \otimes f_{\dot{J}}^{I} \otimes \Xi_{\lambda_{4}}^{\lambda_{3}}$ \\
second & $\bar{\Xi}_{\lambda_{2}}^{\lambda_{1}} \otimes l_{\dot{j}}^{I}$ \\
third & $r_{\dot{J}}^{I^{I}} \otimes \Xi_{\lambda_{2}}^{\lambda_{1}}$ \\
fourth & $\sigma_{J}^{I}$ \\
\hline any & $X, X_{j}^{I}$ or $Y_{\dot{j}}^{I}$ \\
\hline \hline
\end{tabular}

Table 1: Some basis vectors of the grand string algebra. They form an overcomplete set of generators for the open string algebra. $\Lambda$ and $\Lambda_{F}$ are positive integers. $\lambda_{1}, \lambda_{2}, \lambda_{3}$ and $\lambda_{4}$ are positive integers between 1 and $\Lambda_{F}$ inclusive. $\dot{I}$ and $\dot{J}$ are finite empty or non-empty sequences of integers, each of which is between 1 and $\Lambda$ inclusive. $I$ and $J$ are finite non-empty sequences of integers, each of which is between 1 and $\Lambda$ inclusive. $\Xi$ and $\Xi$ are operators acting on two different $\Lambda_{F}$-dimensional Hilbert spaces, and $f, l, r$ and $\sigma$ are operators acting on the same infinite-dimensional Hilbert spaces. All three Hilbert spaces were introduced in Ref. [9]. (There is some abuse of notations here for the sake of future convenience; strictly speaking, the more proper notations $\bar{\Xi}_{\lambda_{2}}^{\lambda_{1}} \otimes l_{j}^{\dot{I}} \otimes 1,1 \otimes r_{\dot{J}}^{\dot{I}} \otimes \Xi_{\lambda_{2}}^{\lambda_{1}}$ and $1 \otimes \sigma_{J}^{I} \otimes 1$ for the operators of the second, third and fourth kind, respectively, refer to the defining representation.)

The elements of the grand string algebra were originated from operators acting on closed or open matrix chains (which are sometimes called closed or open singlet states). Some of them are shown in Table 11. A physical observable is a linear combination of such operators. An operator of the first kind replaces a whole open singlet state with a finite linear combination of open single states; an operator of the second kind replaces the conjugate and the adjacent adjoint partons of an open singlet state with a finite linear combination of open singlet states with possibly other conjugate and adjoint partons; an operator of the third kind is similar to the second kind in action except that it acts on the end with a fundamental parton; an operator of the fourth kind propagates an open singlet state to a finite linear combination of open singlet states in each of which a middle segment of adjacent adjoint 
partons in the original state is replaced with a possibly different sequence of adjoint partons.

Note that as operators acting on closed or open matrix chains, the operators tabulated are not linearly independent; as elements of the grand string algebra, however, they are linearly independent. Listed below are the Lie brackets of the grand string algebra between

1. an operator of the first kind and any operator:

$$
\begin{aligned}
& {\left[\bar{\Xi}_{\lambda_{2}}^{\lambda_{1}} \otimes f_{\dot{J}}^{\dot{I}} \otimes \Xi_{\lambda_{4}}^{\lambda_{3}}, \bar{\Xi}_{\lambda_{6}}^{\lambda_{5}} \otimes f_{\dot{L}}^{\dot{K}} \otimes \Xi_{\lambda_{8}}^{\lambda_{7}}\right]=}
\end{aligned}
$$

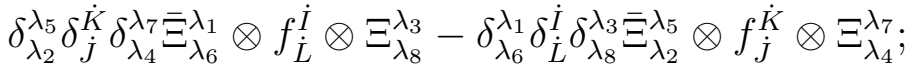

$$
\begin{aligned}
& {\left[\bar{\Xi}_{\lambda_{2}}^{\lambda_{1}} \otimes f_{\dot{J}}^{\dot{I}} \otimes \Xi_{\lambda_{4}}^{\lambda_{3}}, \bar{\Xi}_{\lambda_{6}}^{\lambda_{5}} \otimes l_{\dot{L}}^{\dot{K}}\right]=} \\
& \delta_{\lambda_{2}}^{\lambda_{5}} \bar{\Xi}_{\lambda_{6}}^{\lambda_{1}} \otimes \sum_{\dot{J}_{1} \dot{J}_{2}=\dot{J}} \delta_{\dot{J}_{1}}^{\dot{K}} f_{\dot{L} \dot{J}_{2}}^{\dot{I}} \otimes \Xi_{\lambda_{4}}^{\lambda_{3}}-\delta_{\lambda_{6}}^{\lambda_{1}} \bar{\Xi}_{\lambda_{2}}^{\lambda_{5}} \otimes \sum_{\dot{I}_{1} \dot{I}_{2}=\dot{I}} \delta_{\dot{L}}^{\dot{I}_{1}} f_{\dot{J}}^{\dot{K} \dot{I}_{2}} \otimes \Xi_{\lambda_{4}}^{\lambda_{3}} ; \\
& {\left[\bar{\Xi}_{\lambda_{2}}^{\lambda_{1}} \otimes f_{\dot{J}}^{\dot{I}} \otimes \Xi_{\lambda_{4}}^{\lambda_{3}}, r_{\dot{L}}^{\dot{K}} \otimes \Xi_{\lambda_{6}}^{\lambda_{5}}\right]=} \\
& \delta_{\lambda_{4}}^{\lambda_{5}} \bar{\Xi}_{\lambda_{2}}^{\lambda_{1}} \otimes \sum_{\dot{J}_{1} j_{2}=\dot{J}} \delta_{\dot{J}_{2}}^{\dot{K}} f_{\dot{J}_{1} \dot{L}}^{\dot{I}} \otimes \Xi_{\lambda_{6}}^{\lambda_{3}}-\delta_{\lambda_{6}}^{\lambda_{3}} \bar{\Xi}_{\lambda_{2}}^{\lambda_{1}} \otimes \sum_{\dot{I}_{1} \dot{I}_{2}=\dot{I}} \delta_{\dot{L}}^{\dot{I}_{2}} f_{\dot{J}}^{\dot{I}_{1} \dot{K}} \otimes \Xi_{\lambda_{4}}^{\lambda_{5}} ;(3
\end{aligned}
$$

and

$$
\begin{aligned}
& {\left[\bar{\Xi}_{\lambda_{2}}^{\lambda_{1}} \otimes f_{\dot{J}}^{\dot{I}} \otimes \Xi_{\lambda_{4}}^{\lambda_{3}}, \sigma_{L}^{K}\right]=} \\
& \quad \bar{\Xi}_{\lambda_{2}}^{\lambda_{1}} \otimes\left(\sum_{\dot{J}_{1} J_{2} \dot{J}_{3}=\dot{J}} \delta_{J_{2}}^{K} f_{\dot{J}_{1} L \dot{J}_{3}}^{\dot{I}}-\sum_{\dot{I}_{1} I_{2} \dot{I}_{3}=\dot{I}} \delta_{L}^{I_{2}} f_{\dot{J}}^{\dot{I}_{1} K \dot{I}_{3}}\right) \otimes \Xi_{\lambda_{4}}^{\lambda_{3}}
\end{aligned}
$$

2. an operator of the second kind and an operator of the second, third or fourth kind:

$$
\begin{aligned}
& {\left[\bar{\Xi}_{\lambda_{2}}^{\lambda_{1}} \otimes l_{\dot{J}}^{\dot{I}}, \bar{\Xi}_{\lambda_{4}}^{\lambda_{3}} \otimes l_{\dot{L}}^{\dot{K}}\right]=} \\
& \delta_{\lambda_{2}}^{\lambda_{3}} \bar{\Xi}_{\lambda_{4}}^{\lambda_{1}} \otimes\left(\delta_{\dot{J}}^{\dot{K}} l_{\dot{L}}^{\dot{I}}+\sum_{j_{1} J_{2}=\dot{J}} \delta_{\dot{J}_{1}}^{\dot{K}} l_{\dot{L} J_{2}}^{\dot{I}}+\sum_{\dot{K}_{1} K_{2}=\dot{K}} \delta_{\dot{J}}^{\dot{K}_{1}} l_{\dot{L}}^{\dot{I} K_{2}}\right) \\
& -\delta_{\lambda_{4}}^{\lambda_{1}} \bar{\Xi}_{\lambda_{2}}^{\lambda_{3}} \otimes\left(\delta_{\dot{L}}^{\dot{I}} l_{\dot{J}}^{\dot{K}}+\sum_{\dot{L}_{1} L_{2}=\dot{L}} \delta_{\dot{L}_{1}}^{\dot{I}} l_{\dot{J} L_{2}}^{\dot{K}}+\sum_{\dot{I}_{1} I_{2}=\dot{I}} \delta_{\dot{L}}^{\dot{I}_{1}} l_{\dot{J}}^{\dot{K} I_{2}}\right)
\end{aligned}
$$




$$
\begin{aligned}
& {\left[\bar{\Xi}_{\lambda_{2}}^{\lambda_{1}} \otimes l_{\dot{J}}^{\dot{I}}, r_{\dot{L}}^{\dot{K}} \otimes \Xi_{\lambda_{4}}^{\lambda_{3}}\right]=} \\
& \bar{\Xi}_{\lambda_{2}}^{\lambda_{1}} \otimes\left(\sum_{\substack{\dot{J}_{1} \dot{J}_{2}=\dot{J} \\
\dot{K}_{1} \dot{K}_{2}=\dot{K}}} \delta_{\dot{J}_{2}}^{\dot{K}_{1}} f_{\dot{J}_{1} \dot{L}}^{\dot{I} \dot{K}_{2}}-\sum_{\substack{\dot{I}_{1} \dot{I}_{2}=\dot{I} \\
\dot{L}_{1} \dot{L}_{2}=\dot{L}}} \delta_{\dot{L}_{1}}^{\dot{I}_{2}} f_{\dot{J} \dot{L}_{2}}^{\dot{I}_{1} \dot{K}}\right) \otimes \Xi_{\lambda_{4}}^{\lambda_{3}} ;
\end{aligned}
$$

and

$$
\begin{aligned}
& {\left[\bar{\Xi}_{\lambda_{2}}^{\lambda_{1}} \otimes l_{\dot{J}}^{\dot{I}}, \sigma_{L}^{K}\right]=} \\
& \bar{\Xi}_{\lambda_{2}}^{\lambda_{1}} \otimes\left(\delta_{\dot{J}}^{K} l_{L}^{\dot{I}}+\sum_{K_{1} K_{2}=K} \delta_{\dot{J}}^{K_{1}} l_{L}^{\dot{I} K_{2}}+\sum_{J_{1} J_{2}=\dot{J}} \delta_{J_{2}}^{K} l_{J_{1} L}^{\dot{I}}\right. \\
& +\sum_{J_{1} J_{2}=\dot{J}} \delta_{J_{1}}^{K} l_{L J_{2}}^{\dot{I}}+\sum_{J_{1} J_{2}=\dot{J}} \delta_{J_{2}}^{K_{1}} l_{J_{1} L}^{\dot{I} K_{2}}+\sum_{J_{1} J_{2} J_{3}=\dot{J}} \delta_{J_{2}}^{K} l_{J_{1} L J_{3}}^{\dot{I}} \\
& K_{1} K_{2}=K \\
& -\delta_{L}^{\dot{I}} l_{\dot{J}}^{K}-\sum_{L_{1} L_{2}=L} \delta_{L_{1}}^{\dot{I}} l_{\dot{J} L_{2}}^{K}-\sum_{I_{1} I_{2}=\dot{I}} \delta_{L}^{I_{2}} l_{\dot{J}}^{I_{1} K} \\
& \left.-\sum_{I_{1} I_{2}=\dot{I}} \delta_{L}^{I_{1}} l_{\dot{j}}^{K I_{2}}-\sum_{\substack{L_{1} L_{2}=L \\
I_{1} I_{2}=\dot{I}}} \delta_{L_{1}}^{I_{2}} l_{\dot{J} L_{2}}^{I_{1} K}-\sum_{I_{1} I_{2} I_{3}=\dot{I}} \delta_{L}^{I_{2}} l_{\dot{J}}^{I_{1} K I_{3}}\right) .
\end{aligned}
$$

3. an operator of the third kind and an operator of the third or fourth kind:

$$
\begin{aligned}
& {\left[\begin{array}{l}
r_{\dot{J}}^{\dot{I}} \otimes \Xi_{\lambda_{2}}^{\lambda_{1}}, r_{\dot{L}}^{\dot{K}} \otimes \Xi_{\lambda_{4}}^{\lambda_{3}} \\
\end{array} \quad \delta_{\lambda_{2}}^{\lambda_{3}}\left(\delta_{\dot{J}}^{\dot{K}} r_{\dot{L}}^{\dot{I}}+\sum_{J_{1} \dot{J}_{2}=\dot{J}} \delta_{\dot{J}_{2}}^{\dot{K}} r_{J_{1} \dot{L}}^{\dot{I}}+\sum_{K_{1} \dot{K}_{2}=\dot{K}} \delta_{\dot{J}}^{\dot{K}_{2}} r_{\dot{L}}^{K_{1} \dot{I}}\right) \otimes \Xi_{\lambda_{4}}^{\lambda_{1}}\right.} \\
& \quad-\delta_{\lambda_{4}}^{\lambda_{1}}\left(\delta_{\dot{L}}^{\dot{I}} r_{\dot{J}}^{\dot{K}}+\sum_{L_{1} \dot{L}_{2}=\dot{L}} \delta_{\dot{L}_{2}}^{\dot{I}} r_{L_{1} \dot{J}}^{\dot{K}}+\sum_{I_{1} \dot{I}_{2}=\dot{I}} \delta_{\dot{L}}^{\dot{I}_{2}} r_{\dot{j}}^{I_{1} \dot{K}}\right) \otimes \Xi_{\lambda_{2}}^{\lambda_{3}} \text { and }
\end{aligned}
$$




$$
\begin{aligned}
& {\left[r_{\dot{J}}^{\dot{I}} \otimes \Xi_{\lambda_{2}}^{\lambda_{1}}, \sigma_{L}^{K}\right]=} \\
& \left(\delta_{\dot{J}}^{K} r_{L}^{\dot{I}}+\sum_{K_{1} K_{2}=K} \delta_{\dot{J}}^{K_{2}} r_{L}^{K_{1} \dot{I}}+\sum_{J_{1} J_{2}=\dot{J}} \delta_{J_{2}}^{K} r_{J_{1} L}^{\dot{I}}+\sum_{J_{1} J_{2}=\dot{J}} \delta_{J_{1}}^{K} r_{L J_{2}}^{\dot{I}}\right. \\
& +\sum_{J_{1} J_{2}=\dot{J}} \delta_{J_{1}}^{K_{2}} r_{L J_{2}}^{K_{1} \dot{I}}+\sum_{J_{1} J_{2} J_{3}=\dot{J}} \delta_{J_{2}}^{K} r_{J_{1} L J_{3}}^{\dot{I}} \\
& K_{1} K_{2}=K \\
& -\delta_{L}^{\dot{I}} r_{\dot{J}}^{K}-\sum_{L_{1} L_{2}=L} \delta_{L_{2}}^{\dot{I}} r_{L_{1} \dot{J}}^{K}-\sum_{I_{1} I_{2}=\dot{I}} \delta_{L}^{I_{2}} r_{\dot{J}}^{I_{1} K}-\sum_{I_{1} I_{2}=\dot{I}} \delta_{L}^{I_{1}} r_{\dot{J}}^{K I_{2}} \\
& \left.-\sum_{\substack{L_{1} L_{2}=L \\
I_{1} I_{2}=\dot{I}}} \delta_{L_{2}}^{I_{1}} r_{L_{1} \dot{J}}^{K I_{2}}-\sum_{I_{1} I_{2} I_{3}=\dot{I}} \delta_{L}^{I_{2}} r_{\dot{j}}^{I_{1} K I_{3}}\right) \otimes \Xi_{\lambda_{2}}^{\lambda_{1}} \text {. }
\end{aligned}
$$

4. two operators of the fourth kind:

$$
\begin{aligned}
& {\left[\sigma_{J}^{I}, \sigma_{L}^{K}\right]=\delta_{J}^{K} \sigma_{L}^{I}+\sum_{J_{1} J_{2}=J} \delta_{J_{2}}^{K} \sigma_{J_{1} L}^{I}+\sum_{K_{1} K_{2}=K} \delta_{J}^{K_{1}} \sigma_{L}^{I K_{2}}} \\
& +\sum_{J_{1} J_{2}=J} \delta_{J_{2}}^{K_{1}} \sigma_{J_{1} L}^{I K_{2}}+\sum_{J_{1} J_{2}=J} \delta_{J_{1}}^{K} \sigma_{L J_{2}}^{I}+\sum_{K_{1} K_{2}=K} \delta_{J}^{K_{2}} \sigma_{L}^{K_{1} I} \\
& +\sum_{J_{1} K_{2}=K} \delta_{J_{1}=J}^{K_{2}} \sigma_{L J_{2}}^{K_{1} I}+\sum_{J_{1} J_{2} J_{3}=J} \delta_{J_{2}}^{K} \sigma_{J_{1} L J_{3}}^{I}+\sum_{K_{1} K_{2} K_{3}=K} \delta_{J}^{K_{2}} \sigma_{L}^{K_{1} I K_{3}} \\
& \\
& \quad-(I \leftrightarrow K, J \leftrightarrow L)+\cdots,
\end{aligned}
$$

The ellipses in the last equation represent terms which cannot be written in terms of the operators listed in Table 1; they play no role in the open string algebra, to be introduced immediately.

As the elements of the grand string algebra come from phyiscal observables of open matrix chains, it should not be surprising the open matrix chains provide a representation of the grand string algebra, albeit not a 
faithful one. As we mentioned in the Introduction, an open matrix chain can be abstractly written as $\bar{\phi}^{\lambda_{1}} \otimes s^{\dot{K}} \otimes \phi^{\lambda_{2}} . \bar{\phi}^{1}, \bar{\phi}^{2}, \ldots$, and $\bar{\phi}^{\Lambda_{F}}$ span a $\Lambda_{F^{-}}$ dimensional vector space; $\phi^{1}, \phi^{2}, \ldots$, and $\phi^{\Lambda_{F}}$ span another $\Lambda_{F}$-dimensional vector space; and all vectors of the form $s^{\dot{K}}$ span an infinite-dimensional vector space. Let $\mathcal{T}_{o}$ be the vector space consisting of finite linear combinations of open matrix chains. The actions of the four kinds of operators on an open matrix chain are given by

$$
\begin{aligned}
\bar{\Xi}_{\lambda_{2}}^{\lambda_{1}} \otimes f_{\dot{J}}^{\dot{I}} \otimes \Xi_{\lambda_{4}}^{\lambda_{3}}\left(\bar{\phi}^{\lambda_{5}} \otimes s^{\dot{K}} \otimes \phi^{\lambda_{6}}\right) & =\delta_{\lambda_{2}}^{\lambda_{5}} \delta_{\dot{J}}^{\dot{K}} \delta_{\lambda_{4}}^{\lambda_{6}} \bar{\phi}^{\lambda_{1}} \otimes s^{\dot{I}} \otimes \phi^{\lambda_{3}} \\
\bar{\Xi}_{\lambda_{2}}^{\lambda_{1}} \otimes l_{\dot{J}}^{\dot{I}}\left(\bar{\phi}^{\lambda_{3}} \otimes s^{\dot{K}} \otimes \phi^{\lambda_{4}}\right) & =\delta_{\lambda_{2}}^{\lambda_{3}} \sum_{\dot{K}_{1} \dot{K}_{2}=\dot{K}} \delta_{\dot{J}}^{\dot{K}_{1}} \bar{\phi}^{\lambda_{1}} \otimes s^{\dot{I} \dot{K}_{2}} \otimes \phi^{\lambda_{1}}(1) \\
r_{\dot{J}}^{\dot{I}} \otimes \Xi_{\lambda_{2}}^{\lambda_{1}}\left(\bar{\phi}^{\lambda_{3}} \otimes s^{\dot{K}} \otimes \phi^{\lambda_{4}}\right) & =\delta_{\lambda_{2}}^{\lambda_{4}} \sum_{\dot{K}_{1} \dot{K}_{2}=\dot{K}} \delta_{\dot{J}}^{\dot{K}_{2}} \bar{\phi}^{\lambda_{3}} \otimes s^{\dot{K} \dot{K}_{1}} \otimes \phi^{\lambda_{1}}(13)
\end{aligned}
$$

and

$$
\sigma_{J}^{I}\left(\bar{\phi}^{\lambda_{1}} \otimes s^{\dot{K}} \otimes \phi^{\lambda_{2}}\right)=\bar{\phi}^{\lambda_{1}} \otimes\left(\sum_{\dot{K}_{1} K_{2} \dot{K}_{3}=\dot{K}} \delta_{J}^{K_{2}} s^{\dot{K}_{1} I \dot{K}_{3}}\right) \otimes \phi^{\lambda_{2}} .
$$

$\mathcal{T}_{o}$ is a representation space for the grand string algebra.

Definition 2.1 The Lie algebra denoted as $\hat{G}_{\Lambda, \Lambda_{F}}$ in Ref.[9] and later on called the open string algebra in Ref.[12] is defined as the quotient of the grand string algebra by the annihilator of the representation $\mathcal{T}_{o}$. We will call $\mathcal{T}_{o}$ the defining representation.

Eqs.(11), (5), (8) and (10) show that the space generated by each kind of operators forms a subalgebra of the open string algebra. The four subalgebras were denoted by $F_{\Lambda, \Lambda_{F}}=g l\left(\Lambda_{F}\right) \otimes F_{\Lambda} \otimes g l\left(\Lambda_{F}\right), g l\left(\Lambda_{F}\right) \otimes \hat{L}_{\Lambda}, \hat{R}_{\Lambda} \otimes g l\left(\Lambda_{F}\right)$ and $\hat{\Sigma}_{\Lambda}$, respectively, in Ref.[9]. In addition, Eqs.(11) to (4) tell us that $g l\left(\Lambda_{F}\right) \otimes F_{\Lambda} \otimes g l\left(\Lambda_{F}\right)$ is a proper ideal isomorphic to $g l(\infty)$, and Eqs.(11) to (9) tell us that all the operators of the first three kinds together span a bigger proper ideal $\hat{M}_{\Lambda, \Lambda_{F}}$.

For future convenience, let us introduce some more operators of the fourth kind acting on the defining representation space. They are $\sigma_{\emptyset}^{\emptyset}, \sigma_{\emptyset}^{I}$ and $\sigma_{J}^{\emptyset}$, and are defined by

$$
\sigma_{\emptyset}^{\emptyset}\left(\bar{\phi}^{\lambda_{1}} \otimes s^{\dot{K}} \otimes \phi^{\lambda_{2}}\right) \equiv(\#(\dot{K})+1) \bar{\phi}^{\lambda_{1}} \otimes s^{\dot{K}} \otimes \phi^{\lambda_{2}},
$$




$$
\sigma_{\emptyset}^{I}\left(\bar{\phi}^{\lambda_{1}} \otimes s^{\dot{K}} \otimes \phi^{\lambda_{2}}\right) \equiv \sum_{\dot{K}_{1} \dot{K}_{2}=\dot{K}} \bar{\phi}^{\lambda_{1}} \otimes s^{\dot{K}_{1} I \dot{K}_{2}} \otimes \phi^{\lambda_{2}}
$$

and

$$
\sigma_{J}^{\emptyset}\left(\bar{\phi}^{\lambda_{1}} \otimes s^{\dot{K}} \otimes \phi^{\lambda_{2}}\right) \equiv \sum_{\dot{K}_{1} K_{2} \dot{K}_{3}=\dot{K}} \delta_{J}^{K_{2}} \bar{\phi}^{\lambda_{1}} \otimes s^{\dot{K}_{1} \dot{K}_{3}} \otimes \phi^{\lambda_{2}}
$$

Though these operators look completely new, they are actually elements of the open string algebra, as can be seen from the following identities which are now fully general:

$$
\begin{aligned}
\sigma_{\dot{J}}^{\dot{I}} & =\sum_{i=1}^{\Lambda} \sigma_{i \dot{J}}^{i \dot{I}}+\sum_{\lambda=1}^{\Lambda_{F}} \bar{\Xi}_{\lambda}^{\lambda} \otimes l_{\dot{J}}^{\dot{I}} \\
& =\sum_{j=1}^{\Lambda} \sigma_{\dot{J} j}^{\dot{I} j}+\sum_{\lambda=1}^{\Lambda_{F}} r_{j}^{\dot{I}} \otimes \Xi_{\lambda}^{\lambda}
\end{aligned}
$$

The reader can check the validity of Eq.(18) by verifying that the left and right hand sides have the same action on any open matrix chain.

Without recourse to Eq.(18), there is a representation of $\sigma_{J}^{\emptyset}$ directly in terms of matrix annihilation operators as shown in the following formula, where the summation convention for color indices is adopted:

$$
\sigma_{J}^{\emptyset}=\frac{1}{N^{(b-2) / 2}} a_{\nu_{b}}^{\nu_{b-1}}\left(j_{b}\right) a_{\nu_{b-1}}^{\nu_{b-2}}\left(j_{b-1}\right) \cdots a_{\nu_{1}}^{\nu_{b}}\left(j_{1}\right)
$$

We know of no representation of $\sigma_{\emptyset}^{\emptyset}$ or $\sigma_{\emptyset}^{I}$ in terms of matrix annihilation or creation operators without using Eq.(18).

Sometimes we will use the generic notation $X_{j}^{\dot{I}}$ or $Y_{\dot{j}}^{\dot{I}}$ to refer to $\bar{\Xi}_{\lambda_{2}}^{\lambda_{1}} \otimes$ $f_{\dot{J}}^{\dot{I}} \otimes \Xi_{\lambda_{4}}^{\lambda_{3}}, \bar{\Xi}_{\lambda_{2}}^{\lambda_{1}} \otimes l_{\dot{j}}^{\dot{I}}, r_{\dot{j}}^{\dot{I}} \otimes \Xi_{\lambda_{2}}^{\lambda_{1}}$ or $\sigma_{\dot{j}}^{\dot{I}}$, ignoring $\lambda_{1}, \lambda_{2}, \lambda_{3}$ and $\lambda_{4}$.

\section{Bases}

The operators listed in Table 1 do not form a basis for the open string algebra because they are overcomplete. In this section, we will work out two bases which will be of use in future discussions. Readers who are not interested in the details may read only the statements of Propositions 3.3 and 3.6, and then move on directly to the next section. 
Before we start, we need to recall a lexicographic ordering for integer sequences from Ref. [12]. We will use it to construct another one for a basis of the open string algebra. (Both orderings are denoted as $>$ as there is no danger of confusion.)

Definition 3.1 We designate $\dot{I}>\dot{J}$ if either

1. $\#(\dot{I})>\#(\dot{J})$; or

2. $\#(\dot{I})=\#(\dot{J})=a \neq 0$, and there exists an integer $r \leq a$ such that $i_{1}=j_{1}, i_{2}=j_{2}, \ldots, i_{r-1}=j_{r-1}$ and $i_{r}>j_{r}$.

Definition 3.2 Here is a lexicographic ordering for a basis of the open string algebra.

1. $X_{\dot{J}}^{\dot{I}}>Y_{\dot{L}}^{\dot{K}}$ if

(a) $\#(\dot{I})-\#(\dot{J})>\#(\dot{K})-\#(\dot{L})$; or

(b) $\#(\dot{I})-\#(\dot{J})=\#(\dot{K})-\#(\dot{L})$ and $\#(\dot{I})+\#(\dot{J})>\#(\dot{K})+\#(\dot{L})$; or

(c) $\#(\dot{I})=\#(\dot{K})$, \#( $\dot{J})=\#(\dot{L})$ and $\dot{J}>\dot{L}$; or

(d) $\dot{J}=\dot{L}, \#(\dot{I})=\#(\dot{K})$ and $\dot{I}>\dot{K}$;

2. $\sigma_{j}^{\dot{I}}>r_{j}^{\dot{I}} \otimes \Xi_{\lambda_{2}}^{\lambda_{1}}>\bar{\Xi}_{\lambda_{4}}^{\lambda_{3}} \otimes l_{j}^{\dot{I}}>\Xi_{\lambda_{6}}^{\lambda_{5}} \otimes f_{\dot{J}}^{\dot{I}} \otimes \Xi_{\lambda_{8}}^{\lambda_{7}}$;

3. $\bar{\Xi}_{\lambda_{2}}^{\lambda_{1}} \otimes f_{j}^{\dot{I}} \otimes \Xi_{\lambda_{4}}^{\lambda_{3}}>\bar{\Xi}_{\lambda_{6}}^{\lambda_{5}} \otimes f_{j}^{\dot{I}} \otimes \Xi_{\lambda_{8}}^{\lambda_{7}}$ if

(a) $\lambda_{2} \lambda_{4}>\lambda_{6} \lambda_{8}$ as concatenated sequences; or

(b) $\lambda_{2} \lambda_{4}=\lambda_{6} \lambda_{8}$ and $\lambda_{1} \lambda_{3}>\lambda_{5} \lambda_{7}$;

4. $\bar{\Xi}_{\lambda_{2}}^{\lambda_{1}} \otimes l_{j}^{\dot{I}}>\bar{\Xi}_{\lambda_{4}}^{\lambda_{3}} \otimes l_{j}^{\dot{I}}$ if

(a) $\lambda_{2}>\lambda_{4}$; or

(b) $\lambda_{2}=\lambda_{4}$ and $\lambda_{1}>\lambda_{3}$;

5. $r_{j}^{\dot{I}} \otimes \Xi_{\lambda_{2}}^{\lambda_{1}}>r_{j}^{\dot{I}} \otimes \Xi_{\lambda_{4}}^{\lambda_{3}}$ if

(a) $\lambda_{2}>\lambda_{4}$; or 
(b) $\lambda_{2}=\lambda_{4}$ and $\lambda_{1}>\lambda_{3}$.

Note that changing the basis changes the lexicographic ordering also.

Proposition 3.3 The following set $\mathcal{B}_{0}$ of elements forms a basis for the open string algebra:

1. all $\bar{\Xi}_{\lambda_{2}}^{\lambda_{1}} \otimes f_{\dot{J}}^{\dot{I}} \otimes \Xi_{\lambda_{4}}^{\lambda_{3}}$ such that $\lambda_{1}+\lambda_{2}>2$ and $\lambda_{3}+\lambda_{4}>2$;

2. all $\bar{\Xi}_{\lambda_{2}}^{\lambda_{1}} \otimes l_{\dot{J}}^{\dot{I}}$ such that $\lambda_{1} \neq 1$ or $\lambda_{2} \neq 1$;

3. all $r_{j}^{\dot{I}} \otimes \Xi_{\lambda_{2}}^{\lambda_{1}}$ such that $\lambda_{1} \neq 1$ or $\lambda_{2} \neq 1$; and

4. all $\sigma_{\dot{j}}^{\dot{I}}$.

This proposition is a consequence of the following two lemmas.

Lemma $3.4 \mathcal{B}_{0}$ is a linearly independent set.

Proof. We will prove this by ad absurdum. Consider an arbitrary sum $X$ of a finite number of the elements listed in Proposition 3.3. Write down $X$ according to the following

Convention: the numerical coefficient of $\sigma_{\dot{j}}^{\dot{I}}$ in $X$ is written as $c\left(\sigma_{\dot{j}}^{\dot{I}}\right)$. The coefficients of other operators are written similarly. (By definition, only a finite number of the coefficients are non-zero.)

Assume that this sum $X$ is identically equal to zero. There are now several possibilities. Consider first the case in which some $c\left(\sigma_{\dot{j}}^{\dot{I}}\right) \neq 0$ in the sum $X$, which can then be written as

$$
\sum_{i=1}^{p} c\left(\sigma_{\dot{J}_{i}}^{\dot{I}_{i}}\right) \sigma_{\dot{J}_{i}}^{\dot{I}_{i}}+\cdots
$$

where $p$ is a finite positive integer, $\dot{J}_{1}=\dot{J}_{2}=\cdots=\dot{J}_{q}<\dot{J}_{q+1} \leq \cdots \leq \dot{J}_{p}$ for some integer $q \leq p, \dot{I}_{r} \neq \dot{I}_{s}$ for $1 \leq r, s \leq q$ such that $r \neq s$, and the ellipses 
denote terms involving operators of other kinds. Then acting the sum on $\bar{\phi}^{1} \otimes s^{j_{1}} \otimes \phi^{1}$ yields

$$
\sum_{i=1}^{q} c\left(\sigma_{\dot{J}_{1}}^{\dot{I}_{i}}\right) \bar{\phi}^{1} \otimes s^{\dot{I}_{i}} \otimes \phi^{1}+\cdots
$$

where the ellipses consist of terms proportional to $\bar{\phi}^{\lambda_{1}} \otimes s^{\dot{K}} \otimes \phi^{\lambda_{2}}$, where $\lambda_{1}>1$ or $\lambda_{2}>1$. This is manifestly non-zero, a contradiction. Hence there is no operator of the form $\sigma_{\dot{j}}^{\dot{I}}$ in the sum.

Similarly, considering the action of the sum on a state of the form $\bar{\phi}^{\rho} \otimes$ $s^{\dot{K}} \otimes \phi^{1}$ will rule out the presence of any $\bar{\Xi}_{\lambda_{2}}^{\lambda_{1}} \otimes l_{j}^{\dot{I}}$ in the sum. Then considering $\bar{\phi}^{1} \otimes s^{\dot{K}} \otimes \phi^{\rho}$ will rule out any $r_{\dot{J}}^{\dot{I}} \otimes \Xi_{\lambda_{2}}^{\lambda_{1}}$. Finally, considering $\bar{\phi}^{\rho_{1}} \otimes s^{\dot{K}} \otimes \phi^{\rho_{2}}$ will eliminate all $\bar{\Xi}_{\lambda_{2}}^{\lambda_{1}} \otimes f_{\dot{J}}^{\dot{I}} \otimes \Xi_{\lambda_{4}}^{\lambda_{3}}$. Consequently, no element of $\mathcal{B}_{0}$ can appear in the sum to make it identically zero, and $\mathcal{B}_{0}$ is linearly independent. Q.E.D.

Lemma 3.5 Any element of the open string algebra can be written as a finite sum of the elements listed in Proposition 3.9.

Proof. This follows from the following formulae, which the reader can check one by one by verifying that the actions of the left and right hand sides of any equation below on any open matrix chain are the same:

$$
\begin{aligned}
\Xi_{1}^{1} \otimes l_{\dot{j}}^{\dot{I}} & =\sigma_{\dot{j}}^{\dot{I}}-\sum_{i=1}^{\Lambda} \sigma_{i \dot{j}}^{i \dot{I}}-\sum_{\lambda=2}^{\Lambda_{F}} \bar{\Xi}_{\lambda}^{\lambda} \otimes l_{\dot{j}}^{\dot{I}} ; \\
r_{\dot{J}}^{\dot{I}} \otimes \Xi_{1}^{1} & =\sigma_{\dot{J}}^{\dot{I}}-\sum_{j=1}^{\Lambda} \sigma_{\dot{J} j}^{\dot{I} j}-\sum_{\lambda=2}^{\Lambda_{F}} r_{\dot{J}}^{\dot{I}} \otimes \Xi_{\lambda}^{\lambda} ; \\
\bar{\Xi}_{\lambda_{2}}^{\lambda_{1}} \otimes f_{\dot{J}}^{\dot{I}} \otimes \Xi_{1}^{1} & =\bar{\Xi}_{\lambda_{2}}^{\lambda_{1}} \otimes l_{\dot{J}}^{\dot{I}}-\sum_{j=1}^{\Lambda} \bar{\Xi}_{\lambda_{2}}^{\lambda_{1}} \otimes l_{\dot{j} j}^{\dot{I} j}-\sum_{\lambda_{3}=2}^{\Lambda_{F}} \bar{\Xi}_{\lambda_{2}}^{\lambda_{1}} \otimes f_{\dot{J}}^{\dot{I}} \otimes \Xi_{\lambda_{3}}^{\lambda_{3}} ;
\end{aligned}
$$

where $\lambda_{1} \neq 1$ or $\lambda_{2} \neq 1$;

$$
\bar{\Xi}_{1}^{1} \otimes f_{\dot{J}}^{\dot{I}} \otimes \Xi_{\lambda_{3}}^{\lambda_{2}}=r_{\dot{J}}^{\dot{I}} \otimes \Xi_{\lambda_{3}}^{\lambda_{2}}-\sum_{i=1}^{\Lambda} r_{i \dot{J}}^{i \dot{I}} \otimes \Xi_{\lambda_{3}}^{\lambda_{2}}-\sum_{\lambda_{1}=2}^{\Lambda_{F}} \bar{\Xi}_{\lambda_{1}}^{\lambda_{1}} \otimes f_{\dot{J}}^{\dot{I}} \otimes \Xi_{\lambda_{3}}^{\lambda_{2}}
$$


where $\lambda_{2} \neq 1$ or $\lambda_{3} \neq 1$; and

$$
\begin{aligned}
\bar{\Xi}_{1}^{1} \otimes f_{\dot{J}}^{\dot{I}} \otimes \Xi_{1}^{1}= & \sigma_{\dot{j}}^{\dot{I}}-\sum_{i=1}^{\Lambda} \sigma_{i \dot{j}}^{i \dot{I}}-\sum_{j=1}^{\Lambda} \sigma_{\dot{J} j}^{\dot{I}_{j}}+\sum_{i, j=1}^{\Lambda} \sigma_{i \dot{J} j}^{i \dot{I} j}-\sum_{\lambda=2}^{\Lambda_{F}} \bar{\Xi}_{\lambda}^{\lambda} \otimes l_{\dot{j}}^{\dot{I}} \\
& +\sum_{\lambda=2}^{\Lambda_{F}} \sum_{j=1}^{\Lambda} \bar{\Xi}_{\lambda}^{\lambda} \otimes l_{\dot{J} j}^{\dot{I} j}-\sum_{\lambda=2}^{\Lambda_{F}} r_{\dot{J}}^{\dot{I}} \otimes \Xi_{\lambda}^{\lambda}+\sum_{\lambda=2}^{\Lambda_{F}} \sum_{i=1}^{\Lambda} r_{i \dot{J}}^{i \dot{I}} \otimes \Xi_{\lambda}^{\lambda} \\
& +\sum_{\lambda_{1}, \lambda_{2}=2}^{\Lambda_{F}} \bar{\Xi}_{\lambda_{1}}^{\lambda_{1}} \otimes f_{\dot{J}}^{\dot{I}} \otimes \Xi_{\lambda_{2}}^{\lambda_{2}} .
\end{aligned}
$$

Q.E.D.

We now give a different basis for the open string algebra. We will use it to construct "Verma-like modules".

Proposition 3.6 The following set $\mathcal{B}_{4}$ of elements form a basis for the open string algebra:

1. all $\bar{\Xi}_{\lambda_{2}}^{\lambda_{1}} \otimes f_{\dot{J}}^{\dot{I}} \otimes \Xi_{\lambda_{4}}^{\lambda_{3}}$;

2. all $\bar{\Xi}_{\lambda_{2}}^{\lambda_{1}} \otimes l_{\dot{j}}^{\dot{I}}$ such that the last integers in $\dot{I}$ and $\dot{J}$ are not simultaneously 1 ;

3. all $r_{\emptyset}^{\emptyset} \otimes \Xi_{\lambda_{2}}^{\lambda_{1}}$ such that $\lambda_{1} \neq 1$ or $\lambda_{2} \neq 1$;

4. all $r_{\emptyset}^{I} \otimes \Xi_{\lambda_{2}}^{\lambda_{1}}$ such that $\lambda_{1} \neq 1, \lambda_{2} \neq 1$ or the first integer of I is not 1 ;

5. all $r_{J}^{\emptyset} \otimes \Xi_{\lambda_{2}}^{\lambda_{1}}$ such that $\lambda_{1} \neq 1, \lambda_{2} \neq 1$ or the first integer of $J$ is not 1 ;

6. all $r_{J}^{I} \otimes \Xi_{\lambda_{2}}^{\lambda_{1}}$ such that the first integers in I and $J$ are not simultaneously 1 ;

7. $\sigma_{\emptyset}^{\emptyset}$, all $\sigma_{\emptyset}^{I}$ and all $\sigma_{J}^{\emptyset}$; and

8. all $\sigma_{J}^{I}$ such that the first integers in $I$ and $J$ are not simultaneously 1 and the last integers in $I$ and $J$ are not simultaneously 1 either.

We need a series of lemmas to prove this assertion. 
Lemma 3.7 The following set $\mathcal{B}_{1}$ of elements is linearly independent:

1. all $\Xi_{\lambda_{2}}^{\lambda_{1}} \otimes f_{\dot{J}}^{\dot{I}} \otimes \Xi_{\lambda_{4}}^{\lambda_{3}}$ such that $\lambda_{1}+\lambda_{2}>2$ and $\lambda_{3}+\lambda_{4}>2$;

2. all $\bar{\Xi}_{\lambda_{2}}^{\lambda_{1}} \otimes l_{j}^{\dot{I}}$;

3. all $r_{j}^{\dot{I}} \otimes \Xi_{\lambda_{2}}^{\lambda_{1}}$ such that $\lambda_{1} \neq 1$ or $\lambda_{2} \neq 1$;

4. $\sigma_{\emptyset}^{\emptyset}$, all $\sigma_{\emptyset}^{I}$ and all $\sigma_{J}^{\emptyset}$; and

5. all $\sigma_{J}^{I}$ such that the first integers in $I$ and $J$ are not simultaneously 1 .

Proof. Consider the following set $\mathcal{B}_{1}(n)$ of operators:

1. all $\bar{\Xi}_{\lambda_{2}}^{\lambda_{1}} \otimes f_{j}^{I} \otimes \Xi_{\lambda_{4}}^{\lambda_{3}}$ such that $\lambda_{1}+\lambda_{2}>2$ and $\lambda_{3}+\lambda_{4}>2$;

2. all $\bar{\Xi}_{\lambda_{2}}^{\lambda_{1}} \otimes l_{j}^{\dot{I}}$ such that $\#(\dot{I})+\#(\dot{J})<n$;

3. all $\bar{\Xi}_{\lambda_{2}}^{\lambda_{1}} \otimes l_{j}^{\dot{I}}$ such that $\#(\dot{I})+\#(\dot{J}) \geq n$ and $\lambda_{1}+\lambda_{2}>2$;

4. all $r_{j}^{\dot{I}} \otimes \Xi_{\lambda_{2}}^{\lambda_{1}}$ such that $\lambda_{1} \neq 1$ or $\lambda_{2} \neq 1$;

5. $\sigma_{\emptyset}^{\emptyset}$, all $\sigma_{\emptyset}^{I}$ and all $\sigma_{J}^{\emptyset}$;

6. all $\sigma_{J}^{I}$ such that $\#(I)+\#(J)<n+2$ and the first integers of $I$ and $J$ are not simultaneously 1 ; and

7. all $\sigma_{J}^{I}$ such that $\#(I)+\#(J) \geq n+2$.

Clearly, $\mathcal{B}_{1}(0)=\mathcal{B}_{0}$ and so $\mathcal{B}_{1}(0)$ is a basis for the open string algebra.

Assume that $\mathcal{B}_{1}(p)$ is linearly independent for some non-negative integer $p$. Consider now the case $\mathcal{B}_{1}(p+1)$. The operators belonging to $\mathcal{B}_{1}(p)$ but not to $\mathcal{B}_{1}(p+1)$ are all of $\sigma_{1 \dot{j}}^{1 \dot{j}}$ such that $\#(\dot{I})+\#(\dot{J})=p$, whereas the operators belonging to $\mathcal{B}_{1}(p+1)$ but not to $\mathcal{B}_{1}(p)$ are all of $\bar{\Xi}_{1}^{1} \otimes l_{j}^{I}$ such that $\#(\dot{I})+\#(\dot{J})=p$. Consider any pair of $\dot{I}$ and $\dot{J}$ such that $\#(\dot{I})+\#(\dot{J})=p$. If there is an integer in $\dot{I}$ larger than 1, define $q_{1}$ to be the minimal non-negative integer such that the $(q+1)$-th integer of $\dot{I}$ is larger than 1; otherwise, define $q_{1}$ to be $\#(\dot{I})$. Define $q_{2}$ from the properties of $\dot{J}$ similarly. Let $q$ be the minimum of $q_{1}$ and $q_{2}$. We can then write $\dot{I}=\dot{I}_{1} \dot{I}_{2}$ and $\dot{J}=\dot{I}_{1} \dot{J}_{2}$, where $\dot{I}_{1}$ 
is the number 1 appearing $q$ times, and $\dot{I}_{2}$ or $\dot{J}_{2}$ is empty or starts with an integer larger than 1. From Eq.(20), we have

$$
\begin{aligned}
\sigma_{1 \dot{J}}^{1 \dot{I}} & =\sigma_{\dot{J}_{2}}^{\dot{I}_{2}}-\sum_{i=2}^{\Lambda} \sigma_{i \dot{J}_{2}}^{i \dot{I}_{2}}-\sum_{i=2}^{\Lambda} \sigma_{i 1 \dot{J}_{2}}^{i 1 \dot{I}_{2}}-\cdots-\sum_{i=2}^{\Lambda} \sigma_{i \dot{I}_{1} \dot{J}_{2}}^{i \dot{I}_{\dot{I}_{2}}} \\
& -\sum_{\lambda=1}^{\Lambda_{F}} \bar{\Xi}_{\lambda}^{\lambda} \otimes l_{\dot{J}_{2}}^{\dot{I}_{2}}-\sum_{\lambda=1}^{\Lambda_{F}} \bar{\Xi}_{\lambda}^{\lambda} \otimes l_{1 \dot{J}_{2}}^{1 \dot{I}_{2}}-\cdots-\sum_{\lambda=1}^{\Lambda_{F}} \bar{\Xi}_{\lambda}^{\lambda} \otimes l_{\dot{I}_{1} \dot{J}_{2}}^{\dot{I}_{1} \dot{I}_{2}}
\end{aligned}
$$

Note that $\sigma_{1 \dot{j}}^{1 \dot{I}}$ belongs to $\mathcal{B}_{1}(p)$ but not to $\mathcal{B}_{1}(p+1), \Xi_{1}^{1} \otimes l_{\dot{I}_{1} \dot{J}_{2}}^{\dot{I}_{1} \dot{I}_{2}}$ belongs to $\mathcal{B}_{1}(p+1)$ but not to $\mathcal{B}_{1}(p)$, and all other terms on the right hand side of Eq.(25) belong to both $\mathcal{B}_{1}(p)$ and $\mathcal{B}_{1}(p+1)$. Eq.(25) therefore provides a one-to-one correspondence between the operators belonging to $\mathcal{B}_{1}(p)$ but not to $\mathcal{B}_{1}(p+1)$, and the operators belonging to $\mathcal{B}_{1}(p+1)$ but not to $\mathcal{B}_{1}(p)$. It then follows from the inductive hypothesis at the beginning of this paragraph that $\mathcal{B}_{1}(p+1)$ is linearly independent. As a result, $\mathcal{B}_{1}(n)$ is linearly independent for any non-negative integer value of $n$. Since any element of $\mathcal{B}_{1}$ belongs to $\mathcal{B}_{1}(n)$ for a sufficient large value of $n, \mathcal{B}_{1}$ is linearly independent, too. Q.E.D.

Lemma 3.8 The following set $\mathcal{B}_{2}$ of elements is linearly independent:

1. all $\bar{\Xi}_{\lambda_{2}}^{\lambda_{1}} \otimes f_{\dot{J}}^{\dot{I}} \otimes \Xi_{\lambda_{4}}^{\lambda_{3}}$ such that $\lambda_{1}+\lambda_{2}>2$ and $\lambda_{3}+\lambda_{4}>2$;

2. all $\bar{\Xi}_{\lambda_{2}}^{\lambda_{1}} \otimes l_{\dot{j}}^{\dot{I}}$

3. all $r_{\emptyset}^{\emptyset} \otimes \Xi_{\lambda_{2}}^{\lambda_{1}}$ such that $\lambda_{1} \neq 1$ or $\lambda_{2} \neq 1$;

4. all $r_{\emptyset}^{I} \otimes \Xi_{\lambda_{2}}^{\lambda_{1}}$ such that $\lambda_{1} \neq 1, \lambda_{2} \neq 1$, or the first integer of $I$ is not 1 ;

5. all $r_{J}^{\emptyset} \otimes \Xi_{\lambda_{2}}^{\lambda_{1}}$ such that $\lambda_{1} \neq 1, \lambda_{2} \neq 1$, or the first integer of $J$ is not 1 ;

6. all $r_{J}^{I} \otimes \Xi_{\lambda_{2}}^{\lambda_{1}}$ such that

(a) $\lambda_{1} \neq 1$

(b) $\lambda_{2} \neq 1$ or

(c) the first integers in $I$ and $J$ are not simultaneously 1;

7. $\sigma_{\emptyset}^{\emptyset}$, all $\sigma_{\emptyset}^{I}$ and all $\sigma_{J}^{\emptyset}$; and 
8. all $\sigma_{J}^{I}$ such that the first integers in $I$ and $J$ are not simultaneously 1 , and the last integers in $I$ and $J$ are not simultaneously 1 either.

Proof. This is done by applying Eq.(21) and an inductive argument similar to that in Lemma 3.7 on the set $\mathcal{B}_{2}(n)$ below:

1. all $\bar{\Xi}_{\lambda_{2}}^{\lambda_{1}} \otimes f_{j}^{I} \otimes \Xi_{\lambda_{4}}^{\lambda_{3}}$ such that $\lambda_{1}+\lambda_{2}>2$ and $\lambda_{3}+\lambda_{4}>2$;

2. all $\bar{\Xi}_{\lambda_{2}}^{\lambda_{1}} \otimes l_{\dot{j}}^{\dot{I}}$;

3. all $r_{\emptyset}^{\emptyset} \otimes \Xi_{\lambda_{2}}^{\lambda_{1}}$ such that $\lambda_{1} \neq 1$ or $\lambda_{2} \neq 1$;

4. all $r_{\emptyset}^{I} \otimes \Xi_{\lambda_{2}}^{\lambda_{1}}$ such that $\#(I)<n$ and at least one of the following three conditions holds:

(a) $\lambda_{1} \neq 1$,

(b) $\lambda_{2} \neq 1$ or

(c) the first integer of $I$ is not 1 ;

5. all $r_{\emptyset}^{I} \otimes \Xi_{\lambda_{2}}^{\lambda_{1}}$ such that $\#(I) \geq n$ and at least one of the following two conditions holds:

(a) $\lambda_{1} \neq 1$ or

(b) $\lambda_{2} \neq 1$

6. all $r_{J}^{\emptyset} \otimes \Xi_{\lambda_{2}}^{\lambda_{1}}$ such that $\#(J)<n$ and at least one of the following three conditions holds:

(a) $\lambda_{1} \neq 1$

(b) $\lambda_{2} \neq 1$ or

(c) the first integer of $J$ is not 1 ;

7. all $r_{J}^{\emptyset} \otimes \Xi_{\lambda_{2}}^{\lambda_{1}}$ such that $\#(J) \geq n$ and at least one of the following two conditions holds:

(a) $\lambda_{1} \neq 1$ or

(b) $\lambda_{2} \neq 1$ 
8. all $r_{J}^{I} \otimes \Xi_{\lambda_{2}}^{\lambda_{1}}$ such that $\#(I)+\#(J)<n$ and at least one of the following three conditions holds:

(a) $\lambda_{1} \neq 1$

(b) $\lambda_{2} \neq 1$ or

(c) the first integers of $I$ and $J$ are not 1 simultaneously;

9. all $r_{J}^{I} \otimes \Xi_{\lambda_{2}}^{\lambda_{1}}$ such that $\#(I)+\#(J) \geq n$ and at least one of the following two conditions holds:
(a) $\lambda_{1} \neq 1$ or
(b) $\lambda_{2} \neq 1$;

10. all $\sigma_{\emptyset}^{\emptyset}$, all $\sigma_{\emptyset}^{I}$ and all $\sigma_{J}^{\emptyset}$;

11. all $\sigma_{J}^{I}$ such that $\#(I)+\#(J)<n+2$, the first integers of $I$ and $J$ are not simultaneously 1 , and the last integers of $I$ and $J$ are not simultaneously 1 either; and

12. all $\sigma_{J}^{I}$ such that $\#(I)+\#(J) \geq n+2$ and the first integers of $I$ and $J$ are not simultaneiously 1 .

We invite the reader to work out the detail. Q.E.D.

Lemma 3.9 The following set $\mathcal{B}_{3}$ of elements is linearly independent:

1. all $\bar{\Xi}_{\lambda_{2}}^{\lambda_{1}} \otimes f_{\dot{J}}^{\dot{I}} \otimes \Xi_{\lambda_{4}}^{\lambda_{3}}$ such that $\lambda_{3}+\lambda_{4}>2$;

2. all $\bar{\Xi}_{\lambda_{2}}^{\lambda_{1}} \otimes l_{\dot{j}}^{\dot{I}}$

3. all $r_{\emptyset}^{\emptyset} \otimes \Xi_{\lambda_{2}}^{\lambda_{1}}$ such that $\lambda_{1} \neq 1$ or $\lambda_{2} \neq 1$;

4. all $r_{\emptyset}^{I} \otimes \Xi_{\lambda_{2}}^{\lambda_{1}}$ such that $\lambda_{1} \neq 1, \lambda_{2} \neq 1$ or the first integer of $I$ is not 1 ;

5. all $r_{J}^{\emptyset} \otimes \Xi_{\lambda_{2}}^{\lambda_{1}}$ such that $\lambda_{1} \neq 1, \lambda_{2} \neq 1$ or the first integer of $J$ is not 1 ;

6. all $r_{J}^{I} \otimes \Xi_{\lambda_{2}}^{\lambda_{1}}$ such that the first integers in I and $J$ are not simultaneously 1 ;

7. $\sigma_{\emptyset}^{\emptyset}$, all $\sigma_{\emptyset}^{I}$ and all $\sigma_{J}^{\emptyset}$; and 
8. all $\sigma_{J}^{I}$ such that the first integers in $I$ and $J$ are not simultaneously 1 and the last integers in $I$ and $J$ are not simultaneously 1 either.

Proof. This is done by applying Eq.(23) and an inductive argument similar to that in the Lemma 3.7 on the set $\mathcal{B}_{3}(n)$ below:

1. all $\bar{\Xi}_{\lambda_{2}}^{\lambda_{1}} \otimes f_{\dot{J}}^{\dot{I}} \otimes \Xi_{\lambda_{4}}^{\lambda_{3}}$ such that

(a) $\#(\dot{I})+\#(\dot{J})<n$, and

(b) $\lambda_{3}+\lambda_{4}>2$;

2. all $\bar{\Xi}_{\lambda_{2}}^{\lambda_{1}} \otimes f_{j}^{\dot{I}} \otimes \Xi_{\lambda_{4}}^{\lambda_{3}}$ such that

(a) $\#(\dot{I})+\#(\dot{J}) \geq n$,

(b) $\lambda_{1}+\lambda_{2}>2$, and

(c) $\lambda_{3}+\lambda_{4}>2$;

3. all $\bar{\Xi}_{\lambda_{2}}^{\lambda_{1}} \otimes l_{\dot{j}}^{\dot{I}}$;

4. all $r_{\emptyset}^{\emptyset} \otimes \Xi_{\lambda_{2}}^{\lambda_{1}}$ such that $\lambda_{1} \neq 1$ or $\lambda_{2} \neq 1$;

5. all $r_{\emptyset}^{I} \otimes \Xi_{\lambda_{2}}^{\lambda_{1}}$ such that $\lambda_{1} \neq 1, \lambda_{2} \neq 1$ or the first integer of $I$ is not 1 ;

6. all $r_{J}^{\emptyset} \otimes \Xi_{\lambda_{2}}^{\lambda_{1}}$ such that $\lambda_{1} \neq 1, \lambda_{2} \neq 1$ or the first integer of $J$ is not 1 ;

7. all $r_{J}^{I} \otimes \Xi_{\lambda_{2}}^{\lambda_{1}}$ such that $\#(I)+\#(J) \geq n+2$ and at least one of the following three conditions holds:

(a) $\lambda_{1} \neq 1$

(b) $\lambda_{2} \neq 1$ or

(c) the first integers in $I$ and $J$ are not simultaneously 1 ;

8. all $r_{J}^{I} \otimes \Xi_{\lambda_{2}}^{\lambda_{1}}$ such that $\#(I)+\#(J)<n+2$ and the first integers in $I$ and $J$ are not simultaneously 1 ;

9. $\sigma_{\emptyset}^{\emptyset}$, all $\sigma_{\emptyset}^{I}$ and all $\sigma_{J}^{\emptyset}$; and

10. all $\sigma_{J}^{I}$ such that the first integers in $I$ and $J$ are not simultaneously 1 , and the last integers in $I$ and $J$ are not simultaneously 1 either. 
Again we invite the reader to work out the detail. Q.E.D.

Lemma $3.10 \mathcal{B}_{4}$ is a linearly independent set.

Proof. This is done by applying Eq.(22) with $\lambda_{1}$ and $\lambda_{2}$ arbitrary, and an inductive argument similar to that in the Lemma 3.7 on the set $\mathcal{B}_{4}(n)$ below:

1. all $\bar{\Xi}_{\lambda_{2}}^{\lambda_{1}} \otimes f_{\dot{J}}^{\dot{I}} \otimes \Xi_{\lambda_{4}}^{\lambda_{3}}$ such that $\#(\dot{I})+\#(\dot{J})<n$;

2. all $\bar{\Xi}_{\lambda_{2}}^{\lambda_{1}} \otimes f_{\dot{j}}^{\dot{I}} \otimes \Xi_{\lambda_{4}}^{\lambda_{3}}$ such that

(a) $\#(\dot{I})+\#(\dot{J}) \geq n$, and

(b) $\lambda_{3} \neq 1$ or $\lambda_{4} \neq 1$;

3. all $\bar{\Xi}_{\lambda_{2}}^{\lambda_{1}} \otimes l_{\dot{J}}^{\dot{I}}$ such that $\#(\dot{I})+\#(\dot{J})<n+2$ and the last integers in $\dot{I}$ and $\dot{J}$ are not simultaneously 1 ;

4. all $\bar{\Xi}_{\lambda_{2}}^{\lambda_{1}} \otimes l_{\dot{J}}^{\dot{I}}$ such that $\#(\dot{I})+\#(\dot{J}) \geq n+2$;

5. all $r_{\emptyset}^{\emptyset} \otimes \Xi_{\lambda_{2}}^{\lambda_{1}}$ such that $\lambda_{1} \neq 1$ or $\lambda_{2} \neq 1$;

6. all $r_{\emptyset}^{I} \otimes \Xi_{\lambda_{2}}^{\lambda_{1}}$ such that $\lambda_{1} \neq 1, \lambda_{2} \neq 1$ or the first integer of $I$ is not 1 ;

7. all $r_{J}^{\emptyset} \otimes \Xi_{\lambda_{2}}^{\lambda_{1}}$ such that $\lambda_{1} \neq 1, \lambda_{2} \neq 1$ or the first integer of $J$ is not 1 ;

8. all $r_{J}^{I} \otimes \Xi_{\lambda_{2}}^{\lambda_{1}}$ such that the first integers in $I$ and $J$ are not simultaneously 1 ;

9. $\sigma_{\emptyset}^{\emptyset}$, all $\sigma_{\emptyset}^{I}$ and all $\sigma_{J}^{\emptyset}$; and

10. all $\sigma_{J}^{I}$ such that the first integers in $I$ and $J$ are not simultaneously 1 , and the last integers in $I$ and $J$ are not simultaneously 1 either.

Once again we invite the reader to work out the detail. Q.E.D.

Lemma 3.11 Any operator of the first three kinds can be written as a finite linear combination of the elements in $\mathcal{B}_{4}$. 
Proof. This follows from the equations below. They come from Eqs.(20) to (24). From Eqs.(26) to (36) in this and the next lemma, $\dot{K}_{0}$ is the empty sequence, and $\dot{K}_{n}=K_{n}$ is the sequence $11 \ldots 1$ with $n$ integers for $n>0$.

The first equation is

$$
\begin{aligned}
\bar{\Xi}_{\lambda_{2}}^{\lambda_{1}} \otimes l_{\dot{J} K_{n}}^{\dot{I} K_{n}}= & \bar{\Xi}_{\lambda_{2}}^{\lambda_{1}} \otimes l_{\dot{J}}^{\dot{I}}-\sum_{p=0}^{n-1} \sum_{j=2}^{\Lambda} \bar{\Xi}_{\lambda_{2}}^{\lambda_{1}} \otimes l_{\dot{J} \dot{K}_{p} j}^{\dot{I} \dot{K}_{p} j} \\
& -\sum_{p=0}^{n-1} \sum_{\lambda_{3}=1}^{\Lambda_{F}} \bar{\Xi}_{\lambda_{2}}^{\lambda_{1}} \otimes f_{\dot{j} \dot{K}_{p}}^{\dot{I} \dot{K}_{p}} \otimes \Xi_{\lambda_{3}}^{\lambda_{3}},
\end{aligned}
$$

where $\lambda_{1}$ and $\lambda_{2}$ are any positive integers not larger than $\Lambda_{F}, n$ is any positive integer, and $\dot{I}$ and $\dot{J}$ are any integer sequences such that at least one of them is empty or has its last integer larger than 1.

The second equation is

$$
\begin{aligned}
r_{K_{n} \dot{J}}^{K_{n} \dot{I}} \otimes \Xi_{\lambda_{3}}^{\lambda_{2}}= & r_{\dot{J}}^{\dot{I}} \otimes \Xi_{\lambda_{3}}^{\lambda_{2}}-\sum_{p=0}^{n-1} \sum_{i=2}^{\Lambda} r_{i \dot{K}_{p} \dot{J}}^{i \dot{K}_{p} \dot{I}} \otimes \Xi_{\lambda_{3}}^{\lambda_{2}} \\
& -\sum_{p=0}^{n-1} \sum_{\lambda_{1}=1}^{\Lambda_{F}} \Xi_{\lambda_{1}}^{\lambda_{1}} \otimes f_{\dot{K}_{p} \dot{I}}^{\dot{K}_{p} \dot{I}} \otimes \Xi_{\lambda_{3}}^{\lambda_{2}},
\end{aligned}
$$

where $n$ is positive, and

1. if $\dot{I}=\dot{J}=\emptyset$, then $\lambda_{1}+\lambda_{2}>2$;

2. if $\dot{I} \neq \emptyset$ and $\dot{J}=\emptyset$, then the first integer of $\dot{I} \neq 1$ or $\lambda_{2}+\lambda_{3}>2$;

3. if $\dot{I}=\emptyset$ and $\dot{J} \neq \emptyset$, then the first integer of $\dot{J} \neq 1$ or $\lambda_{2}+\lambda_{3}>2$; and

4. if $\dot{I} \neq \emptyset$ and $\dot{J} \neq \emptyset$, then the first integers $\dot{I}$ and $\dot{J}$ cannot be 1 simultaneously.

The third equation is

$$
\begin{aligned}
r_{\dot{K}_{n}}^{\dot{K}_{n}} \otimes \Xi_{1}^{1}= & \sum_{\lambda=1}^{\Lambda_{F}} \bar{\Xi}_{\lambda}^{\lambda} \otimes l_{\emptyset}^{\emptyset}-\sum_{\lambda=2}^{\Lambda_{F}} r_{\emptyset}^{\emptyset} \otimes \Xi_{\lambda}^{\lambda}-\sum_{p=0}^{n-1} \sum_{i=2}^{\Lambda} r_{i \dot{K}_{p}}^{i \dot{K}_{p}} \otimes \Xi_{1}^{1} \\
& -\sum_{p=0}^{n-1} \sum_{\lambda=1}^{\Lambda_{F}} \Xi_{\lambda}^{\lambda} \otimes f_{\dot{K}_{p}}^{\dot{K}_{p}} \otimes \Xi_{1}^{1},
\end{aligned}
$$


where $n$ is any non-negative integer. The fourth equation is

$$
\begin{aligned}
r_{\emptyset}^{1 \dot{I}} \otimes \Xi_{1}^{1}= & \sigma_{\emptyset}^{1 \dot{I}}-\sigma_{\emptyset}^{\dot{I} 1}+\sum_{i=2}^{\Lambda} \sigma_{i}^{i \dot{I} 1}-\sum_{j=2}^{\Lambda} \sigma_{j}^{1 \dot{I} j}+\sum_{\lambda=1}^{\Lambda_{F}} \bar{\Xi}_{\lambda}^{\lambda} \otimes l_{\emptyset}^{\dot{I} 1} \\
& -\sum_{\lambda=2}^{\Lambda_{F}} r_{\emptyset}^{1 \dot{I}} \otimes \Xi_{\lambda}^{\lambda},
\end{aligned}
$$

where $\dot{I}$ is any sequence. The fifth equation is

$$
\begin{aligned}
r_{1 j}^{\emptyset} \otimes \Xi_{1}^{1}= & \sigma_{1 j}^{\emptyset}-\sigma_{\dot{j} 1}^{\emptyset}+\sum_{i=2}^{\Lambda} \sigma_{i j 1}^{i}-\sum_{j=2}^{\Lambda} \sigma_{1 j j}^{j}+\sum_{\lambda=1}^{\Lambda_{F}} \bar{\Xi}_{\lambda}^{\lambda} \otimes l_{j 1}^{\emptyset} \\
& -\sum_{\lambda=2}^{\Lambda_{F}} r_{1 j}^{\emptyset} \otimes \Xi_{\lambda}^{\lambda},
\end{aligned}
$$

where $\dot{J}$ is any sequence. The sixth equation is

$$
\begin{aligned}
r_{K_{n}}^{K_{n} 1 \dot{I}} \otimes \Xi_{1}^{1}= & r_{\emptyset}^{1 \dot{I}} \otimes \Xi_{1}^{1}-\sum_{p=0}^{n-1} \sum_{i=2}^{\Lambda} r_{i \dot{K}_{p}}^{i \dot{K}_{p} 1 \dot{I}} \otimes \Xi_{1}^{1} \\
& -\sum_{p=0}^{n-1} \sum_{\lambda_{1}=1}^{\Lambda_{F}} \Xi_{\lambda_{1}}^{\lambda_{1}} \otimes f_{\dot{K}_{p}}^{\dot{K}_{p} 1 \dot{J}} \otimes \Xi_{1}^{1}
\end{aligned}
$$

where $n$ is any positive integer, $\dot{I}$ is any sequence, and $r_{\emptyset}^{1 \dot{I}} \otimes \Xi_{1}^{1}$ is given by Eq.(29). The last equation is

$$
\begin{aligned}
r_{K_{n} 1 j}^{K_{n}} \otimes \Xi_{1}^{1}= & r_{1 \dot{J}}^{\emptyset} \otimes \Xi_{1}^{1}-\sum_{p=0}^{n-1} \sum_{i=2}^{\Lambda} r_{i \dot{K}_{p} 1 j}^{i \dot{K}_{p}} \otimes \Xi_{1}^{1} \\
& -\sum_{p=0}^{n-1} \sum_{\lambda_{1}=1}^{\Lambda_{F}} \Xi_{\lambda_{1}}^{\lambda_{1}} \otimes f_{\dot{K}_{p} 1 \dot{I}}^{\dot{K}_{p}} \otimes \Xi_{1}^{1},
\end{aligned}
$$

where $n$ is any positive integer, $\dot{J}$ is any sequence, and $r_{1 \dot{J}}^{\emptyset} \otimes \Xi_{1}^{1}$ is given by Eq.(30). Q.E.D.

Lemma 3.12 Any operator of the fourth kind can be written as a finite linear combination of the elements in $\mathcal{B}_{4}$. 
Proof. Firstly, notice that

$$
\sigma_{K_{n} \dot{I}}^{K_{n} \dot{I}}=\sigma_{\dot{J}}^{\dot{I}}-\sum_{p=0}^{n-1} \sum_{i=2}^{\Lambda} \sigma_{i \dot{K}_{p} \dot{J}}^{i \dot{K}_{p} \dot{I}}-\sum_{p=0}^{n-1} \sum_{\lambda=1}^{\Lambda_{F}} \bar{\Xi}_{\lambda}^{\lambda} \otimes l_{\dot{K}_{p} \dot{I}}^{\dot{K}_{j} \dot{I}}
$$

and

$$
\sigma_{\dot{J} K_{n}}^{\dot{I} K_{n}}=\sigma_{\dot{J}}^{\dot{I}}-\sum_{p=0}^{n-1} \sum_{j=2}^{\Lambda} \sigma_{\dot{J} \dot{K}_{p} j}^{\dot{I} \dot{K}_{p} j}-\sum_{p=0}^{n-1} \sum_{\lambda=1}^{\Lambda_{F}} r_{\dot{J} \dot{K}_{p}}^{\dot{I} \dot{K}_{p}} \otimes \Xi_{\lambda}^{\lambda},
$$

where $n$ is a positive integer, and $\dot{I}$ and $\dot{J}$ satisfy one of the following conditions:

1. $\dot{I}=\emptyset, \dot{J} \neq \emptyset$ and the last integer of $\dot{J}$ is not 1 ;

2. $\dot{J}=\emptyset, \dot{I} \neq \emptyset$ and the last integer of $\dot{I}$ is not 1 ; or

3. both $\dot{I}$ and $\dot{J}$ are non-empty, their first integers are not simultaneously 1 , and their last integers are not simultaneously 1 either.

Lemma 3.11 implies that those l's and r's in Eqs.(33) and (34) which do not belong to $\mathcal{B}_{4}$ can be substituted with the ones which do so. Hence the $\sigma$ 's on the left hand sides can be written as finite linear combinations of the elements of $\mathcal{B}_{4}$.

Secondly, consider $\sigma_{K_{n}}^{K_{m}}$, where $m$ and $n$ are possibly different positive integers. It can be written as

$$
\sigma_{K_{n}}^{K_{m}}=\sigma_{\dot{K}_{n-1}}^{\dot{K}_{m-1}}-\sum_{i=2}^{\Lambda} \sigma_{i \dot{K}_{n-1}}^{i \dot{K}_{m-1}}-\sum_{\lambda=1}^{\Lambda_{F}} \bar{\Xi}_{\lambda}^{\lambda} \otimes l_{\dot{K}_{n-1}}^{\dot{K}_{m-1}} .
$$

The second and last terms on the right hand side of this equation can be written as finite linear combinations of the elements of $\mathcal{B}_{4}$ by Eq.(34) and Lemma 3.11, respectively. Thus an inductive argument on $m+n$ implies that any $\sigma_{K_{n}}^{K_{m}}$ can be written as a finite linear combination of the elements of $\mathcal{B}_{4}$.

Lastly, consider any $\sigma_{K_{m} \dot{J} K_{n}}^{K_{m} \dot{I} K_{n}}$, where $\dot{I}$ and $\dot{J}$ satisfy one of the three conditions just beneath Eq.(34). It can be written as

$$
\sigma_{K_{m} \dot{J} K_{n}}^{K_{m} \dot{I} K_{n}}=\sigma_{\dot{K}_{m-1} \dot{J} K_{n}}^{\dot{K}_{m-1} \dot{I} K_{n}}-\sum_{i=2}^{\Lambda} \sigma_{i \dot{K}_{m-1} \dot{J} K_{n}}^{i \dot{K}_{m-1} \dot{I} K_{n}}-\sum_{\lambda=1}^{\Lambda_{F}} \bar{\Xi}_{\lambda}^{\lambda} \otimes l_{\dot{K}_{m-1} \dot{J} K_{n}}^{\dot{K}_{m-1} \dot{I} K_{n}}
$$


Again, Eq.(34) and Lemma 3.11 show that the second and last terms on the right hand side of this equation can be written as finite linear combinations of the elements of $\mathcal{B}_{4}$. Thus an inductive argument on $\#(\dot{I})+\#(\dot{J})$ implies that these $\sigma$ can be written as finite linear combination of the elements of $\mathcal{B}_{4}$. Q.E.D.

Proposition 3.6 is a direct consequence of Lemmas 3.10, 3.11 and 3.12.

\section{Cartan Subalgebra and Root Vectors}

We are going to work out a Cartan subalgebraf and the root vectors associated with it for the open string algebra. We will need these results in future sections. Once again those who are not interested in details may only read the statements of the propositions in this section, and move on to the next section directly.

Proposition $4.1 A l l \bar{\Xi}_{\lambda_{1}}^{\lambda_{1}} \otimes f_{\dot{I}}^{\dot{I}} \otimes \Xi_{\lambda_{2}}^{\lambda_{2}}$, all $\bar{\Xi}_{\lambda}^{\lambda} \otimes l_{\dot{I}}^{\dot{I}}$, all $r_{\dot{I}}^{\dot{I}} \otimes \Xi_{\lambda}^{\lambda}$ and all $\sigma_{\dot{I}}^{\dot{I}}$ form an overcomplete set of generators of a Cartan subalgebra $G^{00}$ of the open string algebra円.

Proof. In terms of the basis $\mathcal{B}_{0}$, what we need to show is that

1. all $\bar{\Xi}_{\lambda_{1}}^{\lambda_{1}} \otimes f_{\dot{I}}^{\dot{I}} \otimes \Xi_{\lambda_{2}}^{\lambda_{2}}$ such that $\lambda_{1} \neq 1$ and $\lambda_{2} \neq 1$,

2. all $\bar{\Xi}_{\lambda}^{\lambda} \otimes l_{\dot{I}}^{\dot{I}}$, such that $\lambda \neq 1$,

3. all $r_{\dot{I}}^{\dot{I}} \otimes \Xi_{\lambda}^{\lambda}$ such that $\lambda \neq 1$ and

4. all $\sigma_{\dot{I}}^{\dot{I}}$

form a basis for this Cartan subalgebra. It is obvious that $G^{00}$ is commutative and, a fortiori, nilpotent. Consider an element $X$ of the normalizer of $G^{00}$. Let us express $X$ in terms of the basis $\mathcal{B}_{0}$ using the Convention in the proof of Lemma 3.4. Consider

\footnotetext{
${ }^{4}$ Following Humphreys [14], we define a Cartan subalgebra of a Lie algebra $\mathcal{L}$ as a nilpotent subalgebra which is equal to its normalizer in $\mathcal{L}$.

${ }^{5}$ The special case $\Lambda_{F}=1$ has been proven in Ref. [12].
} 
Case 1 There exist in $X$ terms of the form $c\left(\sigma_{\dot{J}_{i}}^{\dot{I}_{i}}\right) \sigma_{\dot{J}_{i}}^{\dot{I}_{i}}$ such that $i$ is a positive integer not larger than $p, \dot{I}_{i} \neq \dot{J}_{i}, c\left(\sigma_{\dot{J}_{i}}^{\dot{I}_{i}}\right) \neq 0$ for each $i$ and $c\left(\sigma_{\dot{J}}^{\dot{I}}\right)=0$ for any other $\dot{I}$ and $\dot{J}$ such that $\dot{I} \neq \dot{I}_{i}$ or $\dot{J} \neq \dot{J}_{i}$ for each $i$. Without loss of generality, we can further assume that either

$$
\dot{I}_{1} \leq \dot{I}_{i} \text { and } \dot{I}_{1} \leq \dot{J}_{i}
$$

for each value of $i$, or

$$
\dot{J}_{1} \leq \dot{I}_{i} \text { and } \dot{J}_{1} \leq \dot{J}_{i}
$$

for each value of $i$.

If Eq.(37) is true, then

$$
\left[\bar{\Xi}_{1}^{1} \otimes f_{\dot{I}_{1}}^{\dot{I}_{1}} \otimes \Xi_{1}^{1}, X\right]=c\left(\sigma_{\dot{J}_{1}}^{\dot{I}_{1}}\right) \bar{\Xi}_{1}^{1} \otimes f_{\dot{J}_{1}}^{\dot{I}_{1}} \otimes \Xi_{1}^{1}+\cdots,
$$

which clearly does not belong to $G^{00}$. If instead Eq.(38) is true, then

$$
\left[\bar{\Xi}_{1}^{1} \otimes f_{\dot{J}_{1}}^{\dot{J}_{1}} \otimes \Xi_{1}^{1}, X\right]=-c\left(\sigma_{\dot{J}_{1}}^{\dot{I}_{1}}\right) \bar{\Xi}_{1}^{1} \otimes f_{\dot{J}_{1}}^{\dot{I}_{1}} \otimes \Xi_{1}^{1}+\cdots,
$$

which clearly does not belong to $G^{00}$ either. Thus there is no term proportional to $\sigma_{\dot{j}}^{\dot{I}}$ in $X$ such that $\dot{I} \neq \dot{J}$.

Next, consider

Case 2 There exist in X terms of the form $c\left(r_{\dot{J}_{i}}^{\dot{I}_{i}} \otimes \Xi_{\rho_{i}}^{\lambda_{i}}\right) r_{\dot{J}_{i}}^{\dot{I}_{i}} \otimes \Xi_{\rho_{i}}^{\lambda_{i}}$ such that the following four conditions hold:

1. $i$ is a positive integer not larger than $p$;

2. $\dot{I}_{i} \lambda_{i} \neq \dot{J}_{i} \rho_{i}$ for each $i$;

3. $c\left(r_{\dot{J}_{i}}^{\dot{I}_{i}} \otimes \Xi_{\rho_{i}}^{\lambda_{i}}\right) \neq 0$ for each $i$; and

4. $c\left(r_{\dot{J}}^{\dot{I}} \otimes \Xi_{\rho}^{\lambda}\right)=0$ for any other $\dot{I}, \dot{J}, \lambda$ and $\rho$ such that $\dot{I} \lambda \neq \dot{I}_{i} \lambda_{i}$ or $\dot{J} \rho \neq \dot{J}_{i} \rho_{i}$ for each $i$. 
Without loss of generality, we can further assume that for all values of $i$, either

$$
\dot{I}_{1} \lambda_{1} \leq \dot{I}_{i} \lambda_{i} \text { and } \dot{I}_{1} \lambda_{1} \leq \dot{J}_{i} \rho_{i}
$$

for each value of $i$, or

$$
\dot{J}_{1} \rho_{1} \leq \dot{I}_{i} \lambda_{i} \text { and } \dot{J}_{1} \rho_{1} \leq \dot{J}_{i} \rho_{i}
$$

for each value of $i$.

If Eq.(41) holds, then

$$
\left[\Xi_{1}^{1} \otimes f_{\dot{I}_{1}}^{\dot{I}_{1}} \otimes \Xi_{\lambda_{1}}^{\lambda_{1}}, X\right]=c\left(r_{\dot{J}_{1}}^{\dot{I}_{1}} \otimes \Xi_{\rho_{1}}^{\lambda_{1}}\right) \Xi_{1}^{1} \otimes f_{\dot{J}_{1}}^{\dot{I}_{1}} \otimes \Xi_{\rho_{1}}^{\lambda_{1}}+\cdots,
$$

which does not belong to $G^{00}$. If instead Eq.(42) holds, then

$$
\left[\bar{\Xi}_{1}^{1} \otimes f_{\dot{J}_{1}}^{\dot{J}_{1}} \otimes \Xi_{\lambda_{1}}^{\lambda_{1}}, X\right]=-c\left(r_{\dot{J}_{1}}^{\dot{I}_{1}} \otimes \Xi_{\rho_{1}}^{\lambda_{1}}\right) \bar{\Xi}_{1}^{1} \otimes f_{\dot{J}_{1}}^{\dot{I}_{1}} \otimes \Xi_{\rho_{1}}^{\lambda_{1}}+\cdots,
$$

which does not belong to $G^{00}$ either. Thus there cannot be any term proportional to $r_{\dot{j}}^{\dot{I}} \otimes \Xi_{\rho}^{\lambda}$ in $X$ such that $\dot{I} \lambda \neq \dot{J} \rho$.

Similar arguments by contradiction enable us to rule out the remaining two cases.

Case 3 There exist in $X$ terms of the form $c\left(\bar{\Xi}_{\rho_{i}}^{\lambda_{i}} \otimes l_{\dot{J}_{i}}^{\dot{I}_{i}}\right) \bar{\Xi}_{\rho_{i}}^{\lambda_{i}} \otimes l_{\dot{J}_{i}}^{\dot{I}_{i}}$ such that the following four conditions hold:

1. $i$ is a positive integer not larger than $p$;

2. $\dot{I}_{i} \lambda_{i} \neq \dot{J}_{i} \rho_{i}$ for each $i$;

3. $c\left(\bar{\Xi}_{\rho_{i}}^{\lambda_{i}} \otimes l_{\dot{J}_{i}}^{\dot{I}_{i}}\right) \neq 0$ for each $i$; and

4. $c\left(\bar{\Xi}_{\rho}^{\lambda} \otimes l_{\dot{J}}^{\dot{I}}\right)=0$ for any other $\dot{I}, \dot{J}, \lambda$ and $\rho$ such that $\dot{I} \lambda \neq \dot{I}_{i} \lambda_{i}$ or $\dot{J} \rho \neq \dot{J}_{i} \rho_{i}$ for each $i$.

Case 4 There exist in $X$ terms of the form $c\left(\bar{\Xi}_{\rho_{i}}^{\lambda_{i}} \otimes f_{\dot{J}_{i}}^{\dot{I}_{i}} \otimes \Xi_{\beta_{i}}^{\alpha_{i}}\right) \bar{\Xi}_{\rho_{i}}^{\lambda_{i}} \otimes f_{\dot{J}_{i}}^{\dot{I}_{i}} \otimes \Xi_{\beta_{i}}^{\alpha_{i}}$ such that the following four conditions hold: 
1. $i$ is a positive integer not larger than $p$;

2. $\dot{I}_{i} \lambda_{i} \alpha_{i} \neq \dot{J} \rho_{i} \beta_{i}$ for each $i$;

3. $c\left(\bar{\Xi}_{\rho_{i}}^{\lambda_{i}} \otimes f_{\dot{J}_{i}}^{\dot{I}_{i}} \otimes \Xi_{\beta_{i}}^{\alpha_{i}}\right) \neq 0$ for each $i$; and

4. $c\left(\bar{\Xi}_{\rho}^{\lambda} \otimes f_{\dot{J}}^{\dot{I}} \otimes \Xi_{\beta}^{\alpha}\right)=0$ for any other $\dot{I}, \dot{J}, \lambda, \rho, \alpha$ and $\beta$ such that $\dot{I} \lambda \alpha \neq \dot{I}_{i} \lambda_{i} \alpha_{i}$ or $\dot{J} \rho \beta \neq \dot{J}_{i} \rho_{i} \beta_{i}$ for each $i$.

Q.E.D.

Proposition 4.2 A necessary and sufficient condition for a vector of the open string algebra to be an eigenvector with respect to the Cartan subalgebra $G^{00}$ is that this vector is proportional to $\bar{\Xi}_{\lambda_{2}}^{\lambda_{1}} \otimes f_{\dot{J}}^{\dot{I}} \otimes \Xi_{\lambda_{4}}^{\lambda_{3}}$, where $\dot{I} \lambda_{1} \lambda_{3} \neq \dot{J} \lambda_{2} \lambda_{4}$ 叫.

Proof. The sufficient part is obvious. Let us prove the necessary part. Write down the eigenvector $V$ in terms of the basis set $\mathcal{B}_{0}$ according to the Convention in the proof of Lemma 3.4. It is clear that $V$ contains no term proportional to an element in $\mathcal{B}_{0}$. Now consider Case 11 in the proof of Proposition 5. If Eq.(37) is true, then Eq.(39) tells us that $V \in F_{\Lambda, \Lambda_{F}}$; if Eq.(38) is true instead, then Eq.(40) still yields the same conclusion that $V \in F_{\Lambda, \Lambda_{F}}$.

Next consider Case 8 in the proof of Proposition 5 together with the additional assumption that $c\left(\sigma_{\dot{J}}^{\dot{I}}\right)=0$ for all $\dot{I}$ and $\dot{J}$. If Eq.(41) is true, then Eq.(43) tells us that $V \in F_{\Lambda, \Lambda_{F}}$; if Eq.(12) is true instead, then Eq.(44) still yields the same conclusion, namely $V \in F_{\Lambda, \Lambda_{F}}$.

Finally, consider Case 3 in the proof of Proposition 5 together with the additional assumptions that $c\left(\sigma_{\dot{J}}^{\dot{I}}\right)=0$ and $c\left(r_{\dot{j}}^{\dot{I}} \otimes \Xi_{\rho}^{\lambda}\right)=0$ for all $\dot{I}, \dot{J}, \lambda$ and $\rho$. An argument similar to the ones in the first two cases will lead us to the same conclusion that $V \in F_{\Lambda, \Lambda_{F}}$.

We therefore conclude that in all case, $V \in F_{\Lambda, \Lambda_{F}}$. Now, we know that $F_{\Lambda, \Lambda_{F}}$ is isomorphic to $g l(\infty)$ whose properties then lead to the necessary part of this proposition. Q.E.D.

\footnotetext{
${ }^{6}$ This is a more elegant proof than the corresponding one in Ref. 12], which deals with the case $\Lambda_{F}=1$ only.
} 


\section{Verma-Like Modules}

Verma modules are a valuable tool for constructing non-trivial unitary lowest weight irreducible representations of familiar Lie algebras like the Virasoro algebra. We are going to adopt the same approach to construct unitary lowest weight irreducible representations for the open string algebra. This algebra, however, differs from the Virasoro algebra in one important aspect - its Cartan subalgebra and the associated root vectors do not span the whole open string algebra. This implies there cannot be any triangular decomposition of the open string algebra in the traditional sense. Nevertheless, there is still a decomposition very similar to the triangular decomposition, and we can use this other decomposition as a starting point to define a module which resembles a Verma module. We will call this a Verma-like module.

It was noted in Ref. 112 that the subalgebra $\hat{\Sigma}_{\Lambda}$ admits a decomposition into subalgebras of "raising", "diagonal" and "lowering" operators. Indeed, we will see shortly that the open string algebra can be $\mathbb{Z}$-graded.

Let $\#(\dot{I})$ be the number of integers in $\dot{I}$, and $\tilde{G}^{m}$ a subspace of the grand string algebra spanned by all operators of any form shown in Table [ 1 (and all operators of the fifth kind not mentioned in Section 21) such that $\#(\dot{I})-\#(\dot{J})=m$ or $\#(I)-\#(J)=m$. Then the grand string algebra is a direct sum of $\tilde{G}^{m}$ for all integral values of $m$. Furthermore, the reader can check from the Lie brackets of the grand string algebra, all of which can be found in Ref.12 and most of which were reproduced in Section 2, that

$$
\left[\tilde{G}^{m}, \tilde{G}^{n}\right] \subseteq \tilde{G}^{m+n}
$$

Hence, the set of all $\tilde{G}^{m}$ provides a $\mathbb{Z}$-grading for the grand string algebra. Moreover, the defining representation is, in a natural way, a graded representation for the grand string algebra with the grade of $\bar{\phi}^{\lambda_{1}} \otimes s^{K} \otimes \phi^{\lambda_{2}}$ equal to $\#(\dot{K})$. Recall from Definition 2.1 that the open string algebra is the quotient of the grand string algebra by the annihilator of this graded representation. It follows that the open string algebra is also $\mathbb{Z}$-graded:

$$
\hat{G}_{\Lambda, \Lambda_{F}}=\bigoplus_{m=-\infty}^{\infty} G^{m}
$$

with $G^{m}$ being the image of $\tilde{G}^{m}$ under the quotient operation and satisfying

$$
\left[G^{m}, G^{n}\right] \subseteq G^{m+n}
$$


The Cartan subalgebra $G^{00}$ is a subalgebra of $G^{0}$. Let $G^{0+}$ be the subspace of $G^{0}$ spanned by all operators of any form shown in Table 1 such that $\dot{I} \lambda_{1} \lambda_{3}>\dot{J} \lambda_{2} \lambda_{4}, \dot{I} \lambda_{1}>\dot{J} \lambda_{2}, \dot{I}>\dot{J}$ or $I>J$. Then $G^{0+}$ is a subalgebra of $G^{0}$ [12]. Likewise, let $G^{0-}$ be the subspace of $G^{0}$ spanned by all operators of any form shown in Table 1 such that $\dot{J} \lambda_{2} \lambda_{4}>\dot{I} \lambda_{1} \lambda_{3}, \dot{J} \lambda_{2}>\dot{I} \lambda_{1}, \dot{J}>\dot{I}$ or $J>I$. Then $G^{0-}$ is another subalgebra of $G^{0}$. Moreover, we have $G^{0}=$ $G^{-} \oplus G^{00} \oplus G^{+}$. Consider

$$
G^{+} \equiv G^{0+} \oplus\left(\bigoplus_{m=1}^{\infty} G^{m}\right)
$$

and

$$
G^{-} \equiv G^{0-} \oplus\left(\bigoplus_{m=-\infty}^{-1} G^{m}\right)
$$

It follows from the fact that $G^{0+}$ is a subalgebra of $G^{0}$ and Eq. (46) that $G^{+}$is a subalgebra of the open string algebra. Likewise, $G^{-}$is another subalgebra of $\hat{G}_{\Lambda, \Lambda_{F}}$. Then

$$
\hat{G}_{\Lambda, \Lambda_{F}}=G^{+} \oplus G^{00} \oplus G^{-}
$$

Let us now construct a module for the open string algebra using Eq.(49). Consider the universal enveloping algebra $\mathcal{U}\left(\hat{G}_{\Lambda, \Lambda_{F}}\right)$ of the open string algebra. Let $h_{I}\left(\lambda_{1} ; \dot{I} ; \lambda_{2}\right), h_{I I}(\lambda ; \dot{I}), h_{I I I}(\dot{I} ; \lambda)$ and $h_{I V}(\dot{I})$ be fixed functions on an integer sequence $\dot{I}$ and, with the exception of $h_{I V}$, the positive integer(s) $\lambda_{1}, \lambda_{2}$ or $\lambda$ also. The subscripts tell us the kinds of operators with which the functions are associated. Construct the left ideal $\mathcal{I}$ of $\mathcal{U}\left(\hat{G}_{\Lambda, \Lambda_{F}}\right)$ generated by

1. all elements in $G^{-}$,

2. all

$$
\bar{\Xi}_{\lambda_{1}}^{\lambda_{1}} \otimes f_{\dot{I}}^{\dot{I}} \otimes \Xi_{\lambda_{2}}^{\lambda_{2}}-h_{I}\left(\lambda_{1} ; \dot{I} ; \lambda_{2}\right) \cdot \mathbf{1}
$$

with 1 being the identity element of $\mathcal{U}\left(\hat{G}_{\Lambda, \Lambda_{F}}\right)$,

3. all

$$
\bar{\Xi}_{\lambda}^{\lambda} \otimes l_{\dot{I}}^{\dot{I}}-h_{I I}(\lambda ; \dot{I}) \cdot \mathbf{1},
$$

such that $\bar{\Xi}_{\lambda}^{\lambda} \otimes l_{\dot{I}}^{\dot{I}} \in \mathcal{B}_{4}$, 
4. all

$$
r_{\dot{I}}^{\dot{I}} \otimes \Xi_{\lambda}^{\lambda}-h_{I I I}(\dot{I} ; \lambda) \cdot \mathbf{1}
$$

such that $r_{\dot{I}}^{\dot{I}} \otimes \Xi_{\lambda}^{\lambda} \in \mathcal{B}_{4}$ and

5. all

$$
\sigma_{\dot{I}}^{\dot{I}}-h_{I V}(\dot{I}) \cdot \mathbf{1}
$$

such that $\sigma_{\dot{I}}^{\dot{I}} \in \mathcal{B}_{4}$.

The values of all $h_{I}, h_{I I}, h_{I I I}$ and $h_{I V}$ listed above can be freely chosen. Fix the values of these four functions on other arguments by the succeeding equations in all of which $\dot{K}_{0}$ stands for the empty sequence and $\dot{K}_{n}=K_{n}$ stands for the sequence $11 \ldots 1$ with $n$ integers for $n>0$ :

$$
\begin{aligned}
h_{I I}\left(\lambda_{1} ; \dot{I} K_{n}\right)= & h_{I I}\left(\lambda_{1} ; \dot{I}\right)-\sum_{p=0}^{n-1} \sum_{j=2}^{\Lambda} h_{I I}\left(\lambda_{1} ; \dot{I} \dot{K}_{p} j\right) \\
& -\sum_{p=0}^{n-1} \sum_{\lambda_{2}=1}^{\Lambda_{F}} h_{I}\left(\lambda_{1} ; \dot{I} \dot{K}_{p} ; \lambda_{2}\right),
\end{aligned}
$$

where $\lambda_{1}$ is any positive integer not larger than $\Lambda_{F}, n$ is any positive integer, and $\dot{I}$ is any integer sequence such that either it is empty or its last integer is larger than 1 (c.f., Eq.(26));

$$
\begin{aligned}
h_{I I I}\left(K_{n} \dot{I} ; \lambda_{2}\right)= & h_{I I I}\left(\dot{I} ; \lambda_{2}\right)-\sum_{p=0}^{n-1} \sum_{i=2}^{\Lambda} h_{I I I}\left(i \dot{K}_{p} \dot{I} ; \lambda_{2}\right) \\
& -\sum_{p=0}^{n-1} \sum_{\lambda_{1}=1}^{\Lambda_{F}} h_{I}\left(\lambda_{1} ; \dot{K}_{p} \dot{I} ; \lambda_{2}\right),
\end{aligned}
$$

where $n$ is positive, and either

1. $\dot{I}$ is empty and $\lambda_{2} \neq 1$, or

2. $\dot{I}$ is non-empty and the first integer of $\dot{I}$ is not 1

(c.f. Eq.(27));

$$
\begin{aligned}
h_{I I I}\left(\dot{K}_{n} ; 1\right)= & \sum_{\lambda=1}^{\Lambda_{F}} h_{I I}(\lambda ; \emptyset)-\sum_{\lambda=2}^{\Lambda_{F}} h_{I I I}(\emptyset ; \lambda)-\sum_{p=0}^{n-1} \sum_{i=2}^{\Lambda} h_{I I I}\left(i \dot{K}_{p} ; 1\right) \\
& -\sum_{p=0}^{n-1} \sum_{\lambda=1}^{\Lambda_{F}} h_{I}\left(\lambda_{1} ; \dot{K}_{p} ; 1\right),
\end{aligned}
$$


where $n$ is any non-negative integer (c.f., Eq.(28));

$$
h_{I V}\left(K_{n} \dot{I}\right)=h_{I V}(\dot{I})-\sum_{p=0}^{n-1} \sum_{i=2}^{\Lambda} h_{I V}\left(i \dot{K}_{p} \dot{I}\right)-\sum_{p=0}^{n-1} \sum_{\lambda=1}^{\Lambda_{F}} h_{I I}\left(\lambda ; \dot{K}_{p} \dot{I}\right),
$$

where $n$ is any positive integer, $\dot{I}$ is either empty or has both its first and last integers larger than 1 , and the values of $h_{I I}$ can either be freely chosen or determined from Eq.(50) (c.f., Eq.(33));

$$
h_{I V}\left(I K_{n}\right)=h_{I V}(I)-\sum_{p=0}^{n-1} \sum_{j=2}^{\Lambda} h_{I V}\left(I \dot{K}_{p} j\right)-\sum_{p=0}^{n-1} \sum_{\lambda=1}^{\Lambda_{F}} h_{I I I}\left(I \dot{K}_{p} ; \lambda\right),
$$

where $n$ is a positive integer, $I$ is a non-empty sequence whose first and last integers are both larger than 1 , and the values of $h_{I I I}$ can either be freely chosen or determined from Eq.(51) or (52) (c.f., Eq.(34));

$$
\begin{aligned}
h_{I V}\left(K_{m} \dot{I} K_{n}\right)= & h_{I V}\left(K_{m} \dot{I}\right)-\sum_{p=0}^{n-1} \sum_{j=2}^{\Lambda} h_{I V}\left(K_{m} \dot{I} \dot{K}_{p} j\right) \\
& -\sum_{p=0}^{n-1} \sum_{\lambda=1}^{\Lambda_{F}} h_{I I I}\left(K_{m} \dot{I} \dot{K}_{p} ; \lambda\right),
\end{aligned}
$$

where both $m$ and $n$ are positive integers, $\dot{I}$ is a non-empty integer sequence whose first and last integers are both larger than 1 , the values of $h_{I I I}$ could be determined from Eq.(51) or (52), and those of $h_{I V}$ could be determined from Eq.(53) (c.f., Eq.(36)). The four functions $h=\left(h_{I}, h_{I I}, h_{I I I}, h_{I V}\right)$ determined in this way will be called a lowest weight. Clearly, the four functions are not linearly indepedent. Eqs.(26), (27), (28), (33), (34) and (36) then imply that $\mathcal{I}$ is spanned by $G^{-}$and all elements of the form

$$
\begin{aligned}
& \bar{\Xi}_{\lambda_{1}}^{\lambda_{1}} \otimes f_{\dot{I}}^{\dot{I}} \otimes \Xi_{\lambda_{2}}^{\lambda_{2}}-h_{I}\left(\lambda_{1} ; \dot{I} ; \lambda_{2}\right) \cdot \mathbf{1}, \\
& \bar{\Xi}_{\lambda}^{\lambda} \otimes l_{\dot{I}}^{\dot{I}}-h_{I I}\left(\lambda_{1} ; \dot{I}\right) \cdot \mathbf{1}, \\
& r_{\dot{I}}^{\dot{I}} \otimes \Xi_{\lambda}^{\lambda}-h_{I I I}(\dot{I} ; \lambda) \cdot \mathbf{1} \text { or } \\
& \sigma_{\dot{I}}^{\dot{I}}-h_{I V}(\dot{I}) \cdot \mathbf{1} .
\end{aligned}
$$

Define $\mathcal{M}$ to be $\mathcal{U} / \mathcal{I}$. $\hat{G}_{\Lambda, \Lambda_{F}}$ acts on $\mathcal{M}$ by left multiplication and so $\mathcal{M}$ is a valid representation of $\hat{G}_{\Lambda, \Lambda_{F}}$. Let $\left|v_{h}\right\rangle$ be the image of $\mathbf{1}$ in $\mathcal{M}$. Then

$$
G^{-}\left|v_{h}\right\rangle=0
$$




$$
\begin{aligned}
\bar{\Xi}_{\lambda_{1}}^{\lambda_{1}} \otimes f_{\dot{I}}^{\dot{I}} \otimes \Xi_{\lambda_{2}}^{\lambda_{2}}\left|v_{h}\right\rangle & =h_{I}\left(\lambda_{1} ; \dot{I} ; \lambda_{2}\right)\left|v_{h}\right\rangle ; \\
\bar{\Xi}_{\lambda}^{\lambda} \otimes l_{\dot{I}}^{\dot{I}}\left|v_{h}\right\rangle & =h_{I I}(\lambda ; \dot{I})\left|v_{h}\right\rangle ; \\
r_{\dot{I}}^{\dot{I}} \otimes \Xi_{\lambda}^{\lambda}\left|v_{h}\right\rangle & =h_{I I I}(\dot{I} ; \lambda)\left|v_{h}\right\rangle ; \text { and } \\
\sigma_{\dot{I}}^{\dot{I}}\left|v_{h}\right\rangle & =h_{I V}(\dot{I})\left|v_{h}\right\rangle .
\end{aligned}
$$

We will call any $\left|v_{h}\right\rangle$ satisfying Eq.(56) a lowest weight vector. (Note that not all elements in $G^{+}$can be written as finite linear combinations of root vectors of $G^{00}$ and so this notion of a lowest weight vector is different from the traditional one.) The Poincaré-Birkhoff-Witt theorem implies that $\left|v_{h}\right\rangle$ together with all the elements in $\mathcal{M}$ of the form

$$
E\left(v_{h}\right)=\prod_{p=1}^{n} X_{j_{p}}^{\dot{I}_{p}}\left|v_{h}\right\rangle,
$$

where $n$ is any positive integer, $X_{j_{p}}^{\dot{I}_{p}} \in G^{+}$for each value of $p$ and the product is arranged in the reverse of the lexicographic ordering in Definition 3.2, forms a basis for $\mathcal{M}$. The expectation value of $E\left(v_{h}\right)$, which we will denote as $\left\langle E\left(v_{h}\right)\right\rangle$, is the coefficient of $\left|v_{h}\right\rangle$ in the expression for $E\left(v_{h}\right)$ written in this basis. We will call $\mathcal{M}$ a Verma-like module. (Again if $G^{+}$and $G^{-}$were spanned by root vectors, $\mathcal{M}$ would be a Verma module.)

A lowest weight representation of the open string algebra is a Vermalike module or a quotient of it]. In general, a lowest weight representation is not irreducible. If there is a maximal subrepresentation of a Verma-like module, the resulting quotient representation will be an irreducible lowest weight representation.

To establish the notion of unitarity for lowest weight representations, we introduce a number of auxiliary notions as follows. Define an antilinear antiinvolution $\omega$ on $\hat{G}_{\Lambda, \Lambda_{F}}$ by

$$
\begin{aligned}
\omega\left(\bar{\Xi}_{\lambda_{2}}^{\lambda_{1}} \otimes f_{\dot{J}}^{\dot{I}} \otimes \Xi_{\lambda_{4}}^{\lambda_{3}}\right) & =\bar{\Xi}_{\lambda_{1}}^{\lambda_{2}} \otimes f_{\dot{I}}^{\dot{J}} \otimes \Xi_{\lambda_{3}}^{\lambda_{4}} ; \\
\omega\left(\bar{\Xi}_{\lambda_{2}}^{\lambda_{1}} \otimes l_{\dot{J}}^{\dot{I}}\right) & =\bar{\Xi}_{\lambda_{1}}^{\lambda_{2}} \otimes l_{\dot{I}}^{\dot{J}} ; \\
\omega\left(r_{\dot{J}}^{\dot{I}} \otimes \Xi_{\lambda_{2}}^{\lambda_{1}}\right) & =r_{\dot{I}}^{\dot{J}} \otimes \Xi_{\lambda_{1}}^{\lambda_{2}} ; \\
\omega\left(\sigma_{J}^{I}\right) & =\sigma_{I}^{J} .
\end{aligned}
$$

\footnotetext{
${ }^{7}$ We called it a highest weight representation in Ref. 16 .
} 
(Readers who know how these four kinds of operators were introduced in Refs. [9] and [12] should be aware that this antilinear anti-involution is nothing but the Hermitian conjugation of creation and annihilation operators of partons.) This antilinear anti-involution of $\hat{G}_{\Lambda, \Lambda_{F}}$ extends straightforwardly to an antilinear anti-involution of its universal enveloping algebra $\mathcal{U}\left(\hat{G}_{\Lambda, \Lambda_{F}}\right)$.

From now on, we assume all the weight functions to be real. This allows us to define a sesquilinear form $\langle\cdot \mid \cdot\rangle$ on two elements $E_{1}\left(v_{h}\right)$ and $E_{2}\left(v_{h}\right)$ of $\mathcal{M}$, both of which are of the form Eq.(57), by

$$
\left\langle E_{1}\left(v_{h}\right) \mid E_{2}\left(v_{h}\right)\right\rangle \equiv\left\langle\left(\omega\left(E_{1}\right) E_{2}\right)\left(v_{h}\right)\right\rangle .
$$

Since $\left\langle\omega(E)\left(v_{h}\right)\right\rangle$ is the complex conjugate of $\left\langle E\left(v_{h}\right)\right\rangle,\langle\cdot \mid \cdot\rangle$ is a Hermitian form of $\mathcal{M}$. Moreover, it is clearly contravariant.

A lowest weight representation is unitary if its Hermitian form is positive definite. Of course, a Verma-like module is not unitary in general. Nevertheless, by a judicious choice of weight functions, it is possible to obtain unitary quotient representations with the help of this Hermitian form. In this case we call the Verma-like module unitarizable.

\section{Tensor Products of the Defining Represen- tation}

Recall the defining representation in Section 2. It is unitary and irreducible. More unitary irreducible representations can be obtained from the defining representation by taking its tensor products. Can they be obtained from Verma-like modules? We will answer this question in the form of a theorem. To state it, we need

Definition 6.1 A Verma-like module is approximately finite if its lowest weight function $h$ satisfies the following conditions:

1. $h_{I}\left(\lambda_{1} ; \dot{I} ; \lambda_{2}\right)-h_{I}\left(\lambda_{3} ; \dot{J} ; \lambda_{4}\right)$ is a non-negative integer if $\dot{J} \lambda_{3} \lambda_{4}>\dot{I} \lambda_{1} \lambda_{2}$;

2. $h_{I I}(\lambda ; \dot{I})=\sum_{\dot{I}_{1}, \lambda_{1}} h_{I}\left(\lambda ; \dot{I} \dot{I}_{1} ; \lambda_{1}\right)$;

3. $h_{I I I}(\dot{I} ; \lambda)=\sum_{\lambda_{1}, \dot{I}_{1}} h_{I}\left(\lambda_{1} ; \dot{I} \dot{I} \dot{I} ; \lambda\right) ;$ and

4. $h_{I V}(\dot{I})=\sum_{\lambda_{1}, \dot{I}_{1}, \dot{I}_{2}, \lambda_{2}} h_{I}\left(\lambda_{1} ; \dot{I}_{1} \dot{I} \dot{I}_{2} ; \lambda_{2}\right)$. 
(By convergence and unitarity, only a finite number of summands can be non-zero in each of the last three equations. 阳

Theorem 6.2 The following statements pertaining to a unitary irreducible representation of open string algebra are equivalent:

1. The representation is a tensor product of the defining representation.

2. The representation is the quotient of an approximately finite Verma-like module by its maximal subrepresentation.

3. The representation is the quotient of a Verma-like module in which $h_{I}$, $h_{I I}, h_{I I I}$ and $h_{I V}$ are all non-zero only on a finite number of arguments by its maximal subrepresentation.

4. The representation is the quotient of a Verma-like module in which $h_{I V}$ is non-zero only on a finite number of arguments by its maximal subrepresentation $[$.

Moreover, the maximal subrepresentations in the above statements are the radical of the Hermitian form of the Verma-like module.

There are some interesting physical interpretations of this theorem. In the context of QCD, a tensor product of the defining representation is a space consisting of multiple meson states. Theorem 6.2 thus reflects once again a long-established fact that in the large- $N$ limit, one cannot break an open string into several, or combine several open strings to one [17. Furthermore, the proof of Proposition 5 clearly shows that $G^{00}$ is a maximally commutative subalgebra of $\hat{G}_{\Lambda, \Lambda_{F}}$. We may thus think of $G^{00}$ as a linear space generated by a maximally commuting set of linearly independent quantum observables, of which the lowest weight state is an eigenstate with all its eigenvalues, or quantum numbers, given by the weight functions. If this state has only a finite number of non-zero quantum numbers, any other state generated by it will have a finite number of non-zero quantum numbers, too. Consequently, the above theorem implies that if an eigenstate, lowest weight or not, has

\footnotetext{
${ }^{8}$ This definition is slightly different from the one we gave earlier in Ref. [16]; here we impose the additional condition that $h_{I V}(\emptyset)$ satisfies the last equation.

${ }^{9}$ We thank S. G. Rajeev for suggesting this fourth statement.
} 
only a finite number of non-zero quantum numbers with respect to these quantum observables, then this eigenstate must be a multiple meson state.

Before embarking on the proof of the equivalences, let us make some simple observations which have, among other things, as consequences the statements about the Hermitian form in the theorem.

Lemma 6.3 The maximal subrepresentation of a unitarizable Verma-like module is the radical of the Hermitian form.

Proof. If we quotient out by the radical of the Hermitian form in the Verma module we get a representation with a non-degenerate Hermitian form (still contravariant of course). A priori it might seem possible that this representation could have a proper unitary quotient. However, exactly due to the unitarity assumption, if there exists a non-zero maximal proper invariant subspace $I$ such that the quotient by it is unitary, then in fact the quotient must be equivalent to $I^{\perp}$. But since the space is cyclic, this is possible only if $I=0$ which is a contradiction. Q.E.D.

Lemma 6.4 If for a given weight $h$ there exists a contravariant unitary lowest (or highest) weight module $V_{h}$, then it is unique.

Proof. Let $\left|v_{h}\right\rangle$ denote the lowest weight vector and let $A_{h}$ denote the annihilator of $\left|v_{h}\right\rangle$ in the envelopping algebra $\mathcal{U}$. Then $V_{h} \simeq \mathcal{U} / A_{h}$ and by Lemma 6.3, $A_{h}$ is equal to the set of $Y \in \mathcal{U}$ for which $Y\left|v_{h}\right\rangle=0 \Leftrightarrow\left\langle Y\left(v_{h}\right)\right|$ $\left.Y\left(v_{h}\right)\right\rangle=0$. By contravariance, the latter condition is expressible entirely in terms of the Lie algebra structure and $h$. Q.E.D.

We will now prove Theorem 6.2 by a series of lemmas in which 1., 2., 3 . and 4. stand for the four enumerated statements in Theorem 6.2.

Lemma $6.51 . \Rightarrow 2$.

Proof. First of all we observe that the defining representation $\mathcal{T}_{o}$ is obviously approximately finite. Indeed, it is elementary to verify that the following identities hold in $\mathcal{T}_{o}$

$$
\bar{\Xi}_{\lambda_{2}}^{\lambda_{1}} \otimes l_{\dot{J}}^{\dot{I}}=\sum_{\lambda_{3}=1}^{\Lambda_{F}} \sum_{\dot{K}} \bar{\Xi}_{\lambda_{2}}^{\lambda_{1}} \otimes f_{\dot{J} \dot{K}}^{\dot{I} K} \otimes \Xi_{\lambda_{3}}^{\lambda_{3}},
$$




$$
\begin{aligned}
r_{\dot{J}}^{\dot{I}} \otimes \Xi_{\lambda_{4}}^{\lambda_{3}} & =\sum_{\lambda_{1}=1}^{\Lambda_{F}} \sum_{\dot{K}} \bar{\Xi}_{\lambda_{1}}^{\lambda_{1}} \otimes f_{\dot{K} \dot{J}}^{\dot{K} \dot{I}} \otimes \Xi_{\lambda_{4}}^{\lambda_{3}} \text { and } \\
\sigma_{\dot{J}}^{\dot{I}} & =\sum_{\lambda_{1}, \lambda_{2}=1}^{\Lambda_{F}} \sum_{\dot{K}_{1}, \dot{K}_{2}} \bar{\Xi}_{\lambda_{2}}^{\lambda_{1}} \otimes f_{\dot{K_{1}} \dot{J} \dot{K}_{2}}^{\dot{K}_{1} \dot{I} \dot{K}_{2}} \otimes \Xi_{\lambda_{2}}^{\lambda_{2}}
\end{aligned}
$$

for all $\dot{I}, \dot{J}, \lambda_{1}, \lambda_{2}, \lambda_{3}$, and $\lambda_{4}$. It is clear that the tensor product $\mathcal{T}_{o}^{d}=\mathcal{T}_{o} \otimes$ $\mathcal{T}_{o} \otimes \cdots \otimes \mathcal{T}_{o}$ ( $d$ copies) will have the same property. Furthermore, any Young symmetrizer $c_{\gamma}$ will define an invariant subspace and a non-zero weight vector $v_{\gamma}$ which is annihilated by any subalgebra $g l(N)^{-} \subset g l(\infty) \equiv g l\left(\Lambda_{F}\right) \otimes F_{\Lambda} \otimes$ $g l\left(\Lambda_{F}\right)$.

Let

$$
\gamma=\left(\gamma_{1}, \gamma_{2}, \ldots\right) \text { with } \gamma_{1} \geq \gamma_{2} \geq \cdots \geq \gamma_{n} \geq 0=\gamma_{n+1}=\gamma_{n+2}=\cdots
$$

such that $d=\gamma_{1}+\gamma_{2}+\ldots+\gamma_{n}$. Again, by looking at the subalgebras $g l(N)$ it follows that $c_{\gamma}\left(\mathcal{T}_{o}\right)$ carries an irreducible representation. That $v_{\gamma}$ is annihilated by all of $G^{-}$and forms a one-dimensional representation for $G^{00}$ is equally clear. The lowest weight is given by the formulae

$$
\begin{aligned}
h_{I}(1 ; \emptyset ; 1) & =\gamma_{1}, \\
h_{I}(1 ; \emptyset ; 2) & =\gamma_{2}, \\
\vdots & \\
h_{I}\left(1 ; \emptyset ; \Lambda_{F}\right) & =\gamma_{\Lambda_{F}}, \\
h_{I}(2 ; \emptyset ; 1) & =\gamma_{\Lambda_{F}+1}, \\
\vdots & \\
h_{I}\left(\Lambda_{F} ; \emptyset ; \Lambda_{F}\right) & =\gamma_{\Lambda_{F} \Lambda_{F}}, \\
h_{I}(1 ; 1 ; 1) & =\gamma_{\Lambda_{F} \Lambda_{F}+1}, \\
\vdots & \\
h_{I}\left(1 ; 1 ; \Lambda_{F}\right) & =\gamma_{\Lambda_{F} \Lambda_{F}+\Lambda_{F}}, \\
h_{I}(2 ; 1 ; 1) & =\gamma_{\Lambda_{F} \Lambda_{F}+\Lambda_{F}+1}, \\
\vdots & \\
h_{I}\left(\Lambda_{F} ; 1 ; \Lambda_{F}\right) & =\gamma_{2 \Lambda_{F} \Lambda_{F}}, \\
h_{I}(1 ; 2 ; 1) & =\gamma_{2 \Lambda_{F} \Lambda_{F}+1},
\end{aligned}
$$




$$
\begin{aligned}
h_{I}\left(\Lambda_{F} ; \Lambda ; \Lambda_{F}\right) & =\gamma_{\Lambda \Lambda_{F} \Lambda_{F}}, \\
h_{I}(1 ; 11 ; 1) & =\gamma_{\Lambda \Lambda_{F} \Lambda_{F}+1}, \\
\vdots & \\
h_{I}\left(\rho_{1} ; \dot{K} ; \rho_{2}\right) & =\gamma_{n} \text { and } \\
h_{I}\left(\lambda_{1} ; \dot{I} ; \lambda_{2}\right) & =0 \text { if } \dot{I} \lambda_{1} \lambda_{2}>\dot{K} \rho_{1} \rho_{2} .
\end{aligned}
$$

Eqs.(60) to (62) clearly also hold in $c_{\gamma}\left(\mathcal{T}_{o}\right)$. Q.E.D.

\section{Lemma 6.6 2. $\Rightarrow 1$.}

Proof. Let $h_{I}$ be given in terms of a $\gamma$ as in Eq.(63). Then, since it is nonzero only on a finite number of arguments, $\gamma$ defines a Young symmetrizer $c_{\gamma}$. Consider $c_{\gamma}\left(\mathcal{T}_{o}\right)$. It is easy to see that this space has the right lowest weight. By Lemma 6.4 it is unique. Q.E.D.

Lemma 6.7 2. $\Rightarrow 3$.

Proof. According to Definition 8, only a finite number of the summands in the formula

$$
h_{I V}(\emptyset)=\sum_{\lambda_{1}, \dot{I}_{1}, \dot{I}_{2}, \lambda_{2}} h_{I}\left(\lambda_{1} ; \dot{I}_{1} \dot{I}_{2} ; \lambda_{2}\right)
$$

are non-zero, so there exists an integer sequence $\dot{K}$ such that $h_{I}\left(\lambda_{1} ; \dot{I} ; \lambda_{2}\right)=0$ for any $\lambda_{1}$ and $\lambda_{2}$ if $\dot{I}>\dot{K}$. Then $h_{I I}(\lambda ; \dot{I})=h_{I I I}(\dot{I} ; \lambda)=h_{I V}(I)=0$ if $\dot{I}>\dot{K}$. In particular, $h_{I I}, h_{I I I}$ and $h_{I V}$ are non-zero on a finite number of arguments only. Q.E.D.

Lemma $6.8 h_{I}\left(\lambda_{2} ; \dot{J} ; \lambda_{4}\right)-h_{I}\left(\lambda_{1} ; \dot{I} ; \lambda_{3}\right)$ is a non-negative integer if $\dot{I} \lambda_{1} \lambda_{3}>$ $\dot{J} \lambda_{2} \lambda_{4}$ 国.

Proof. Let $\dot{I}$ and $\dot{J}$ be arbitrarily chosen integer sequences, and $\lambda_{1}, \lambda_{2}, \lambda_{3}$ and $\lambda_{4}$ arbitrarily chosen positive integers not greater than $\Lambda_{F}$. Notice that

$$
\bar{\Xi}_{\lambda_{2}}^{\lambda_{1}} \otimes f_{\dot{J}}^{\dot{I}} \otimes \Xi_{\lambda_{4}}^{\lambda_{3}}, \bar{\Xi}_{\lambda_{1}}^{\lambda_{1}} \otimes f_{\dot{I}}^{\dot{I}} \otimes \Xi_{\lambda_{3}}^{\lambda_{3}}-\bar{\Xi}_{\lambda_{2}}^{\lambda_{2}} \otimes f_{\dot{J}}^{\dot{J}} \otimes \Xi_{\lambda_{4}}^{\lambda_{4}} \text { and } \bar{\Xi}_{\lambda_{1}}^{\lambda_{2}} \otimes f_{\dot{I}}^{\dot{J}} \Xi_{\lambda_{3}}^{\lambda_{4}},
$$

\footnotetext{
${ }^{10}$ Observe that this result is completely general: any unitary irreducible representation of the open string algebra constructed from a Verma-like module satisfies the first condition in Definition 8.
} 
where $\dot{I} \lambda_{1} \lambda_{3}>\dot{J} \lambda_{2} \lambda_{4}$, span a subalgebra of the open string algebra. This subalgebra is isomorphic to $\operatorname{sl}(2, \mathbb{C})$. We therefore deduce from the representation theory of $\operatorname{sl}(2, \mathbb{C})$ that $h_{I}\left(\lambda_{2} ; \dot{J} ; \lambda_{4}\right)-h_{I}\left(\lambda_{1} ; \dot{I} ; \lambda_{3}\right)$ must be a nonnegative integer. Q.E.D.

Lemma 6.9 3. $\Rightarrow$ 2..

Proof. Since $h_{I}$ is non-zero on a finite number of arguments only, Lemma 10 implies that there exists an integer sequence $\dot{K} \rho_{1} \rho_{2}$ such that

1. $h_{I}\left(\rho_{1} ; \dot{K} ; \rho_{2}\right)>0$;

2. $h_{I}\left(\lambda_{1} ; \dot{I} ; \lambda_{2}\right)=0$ if $\dot{I} \lambda_{1} \lambda_{2}>\dot{K} \rho_{1} \rho_{2} ;$ and

3. $0<h_{I}\left(\lambda_{1} ; \dot{I} ; \lambda_{2}\right) \leq h_{I}\left(\lambda_{3} ; \dot{J} ; \lambda_{4}\right)$ if $\dot{K} \rho_{1} \rho_{2}>\dot{I} \lambda_{1} \lambda_{2}>\dot{J} \lambda_{3} \lambda_{4}$.

In other words, Eq.(63) with the partition $\gamma$ holds.

We will move on to show that $h_{I I}$ satisfies Definition 8. The proofs for $h_{I I I}$ and $h_{I V}$ are similar. Let $\dot{K}_{1} \rho_{3}$ be the integer sequence such that $h_{I I}\left(\rho_{3} ; \dot{K}_{1}\right)>0$ and $h_{I I}(\lambda ; \dot{I})=0$ if $\dot{I} \lambda>\dot{K}_{1} \rho_{3}$. Then Eq. (22) implies that

$$
\sum_{\lambda} h_{I}\left(\rho_{3} ; \dot{K}_{1} ; \lambda\right)=h_{I I}\left(\rho_{3} ; \dot{K}_{1}\right)-\sum_{i} h_{I I}\left(\rho_{3} ; \dot{K}_{1} i\right)>0 .
$$

Hence $\dot{K}_{1} \rho_{3} \leq \dot{K} \rho_{1} . \dot{K}_{1} \rho_{3}<\dot{K} \rho_{1}$ is impossible or else

$$
\sum_{\lambda} h_{I}\left(\rho_{1} ; \dot{K} ; \lambda\right)=h_{I I}\left(\rho_{1} ; \dot{K}\right)-\sum_{i} h_{I I}\left(\rho_{1} ; \dot{K} i\right)=0,
$$

a contradiction. Thus $\rho_{3}=\rho_{1}$ and $\dot{K}_{1}=\dot{K}$. That $h_{I I}$ satisfies Definition 8 now follows from the fact that $h_{I I}(\lambda ; \dot{I})$ is a sum of

1. all $h_{I}\left(\lambda ; \dot{I} \dot{I}_{1} ; \lambda_{1}\right)$ where $\lambda_{1}$ can take on any value and $\dot{I}_{1}$ is an integer sequence such that $\dot{I} \dot{I}_{1} \lambda<\dot{K} \rho_{1}$,

2. all $h_{I I}\left(\lambda ; \dot{I} \dot{I}_{1} i\right)$ where $\dot{I}_{1} i$ is any integer sequence such that $\dot{I} \dot{I}_{1} \lambda<\dot{K} \rho_{1}$ but $\dot{I} \dot{I}_{1} i \lambda>\dot{K} \rho_{1}$

and the fact that the summands in the second family vanish identically. Q.E.D.

Lemma 6.10 3. $\Rightarrow 4$.. 
Proof. Trivial. Q.E.D.

Lemma $6.11 h_{I I}\left(\lambda_{2} ; \dot{J}\right)-h_{I I}\left(\lambda_{1} ; \dot{I}\right) \geq 0$ and $h_{I I I}\left(\dot{J} ; \lambda_{2}\right)-h_{I I I}\left(\dot{I} ; \lambda_{1}\right) \geq 0$ if $\dot{I} \lambda_{1}>\dot{J} \lambda_{2}$.

Proof. This comes from the inequalities

$$
\left\langle v_{h}\left|\left(\bar{\Xi}_{\lambda_{1}}^{\lambda_{2}} \otimes l_{\dot{I}}^{j}\right)\left(\bar{\Xi}_{\lambda_{2}}^{\lambda_{1}} \otimes l_{\dot{J}}^{I}\right)\right| v_{h}\right\rangle \geq 0
$$

and

$$
\left\langle v_{h}\left|\left(r_{\dot{I}}^{\dot{j}} \otimes \Xi_{\lambda_{1}}^{\lambda_{2}}\right)\left(r_{\dot{J}}^{\dot{I}} \otimes \Xi_{\lambda_{2}}^{\lambda_{1}}\right)\right| v_{h}\right\rangle \geq 0 .
$$

Q.E.D.

Lemma 6.12 4. $\Rightarrow 3$. .

Proof. Let $\dot{K}$ be an integer sequence such that $h_{I V}(\dot{K})>0$ and $h_{I V}(\dot{I})=0$ for any $\dot{I}>\dot{K}$. Eqs.(22), (23) and (24) imply that for this $\dot{I}$,

$$
\begin{aligned}
\sum_{\lambda_{1}, \lambda_{2}=1}^{\Lambda_{F}} h_{I}\left(\lambda_{1} ; \dot{I} ; \lambda_{2}\right) & =h_{I V}(\dot{I})-\sum_{i=1}^{\Lambda} h_{I V}(i \dot{I})-\sum_{j=1}^{\Lambda} h_{I V}(\dot{I} j)+\sum_{i, j=1}^{\Lambda} h_{I V}(i \dot{I} j) \\
& =0 .
\end{aligned}
$$

Assume that some $h_{I}\left(\lambda_{1} ; \dot{I} ; \lambda_{2}\right) \neq 0$ in Eq.(64). Then there exist two integers $\rho_{1}$ and $\rho_{2}$ such that $h_{I}\left(\rho_{1} ; \dot{I} ; \rho_{2}\right)<0$. By Lemma 10, $h_{I}\left(\lambda_{3} ; \dot{J} ; \lambda_{4}\right)<0$ if $\dot{J}>\dot{I}$. Hence for this $\dot{J}$,

$$
\begin{aligned}
0 & >\sum_{\lambda_{3}, \lambda_{4}=1}^{\Lambda_{F}} h_{I}\left(\lambda_{3} ; \dot{J} ; \lambda_{4}\right) \\
& =h_{I V}(\dot{J})-\sum_{i=1}^{\Lambda} h_{I V}(i \dot{J})-\sum_{j=1}^{\Lambda} h_{I V}(\dot{J} j)+\sum_{i, j=1}^{\Lambda} h_{I V}(i \dot{J} j) \\
& =0,
\end{aligned}
$$

a contradiction. We thus conclude that $h_{I}\left(\lambda_{1} ; \dot{I} ; \lambda_{2}\right)=0$ for any integer sequence $\dot{I}$ such that $\dot{I}>\dot{K}$ and any integers $\lambda_{1}$ and $\lambda_{2}$. In particular, $h_{I}$ is non-zero on a finite number of arguments only. A similar argument using Lemma 6.11 shows that $h_{I I}$ and $h_{I I I}$ are non-zero on a finite number of arguments only. Q.E.D. 


\section{Other Unitary Irreducible Representations}

Now that we have identified a class of unitary irreducible representations, it is natural for us to ask how other unitary irreducible representations look like. One crucial observation is that not only are the above tensor product representations faithful representations of the full open string algebra, but also they are completely determined by the ideal $s l(\infty)=[g l(\infty), g l(\infty)]$, where $g l(\infty)=F_{\Lambda, \Lambda_{F}}$, and are the only representations that remain faithful and unitary as representations of this ideal. This suggests that other unitary irreducible representations can be obtained as unitary lowest weight representations from the quotient algebra by $s l(\infty)$, i.e. as "truly infinite" (t.i.) representations of the open string algebra - lowest weight representations in which $s l(\infty)$ acts trivially. Indeed, it turns out that

Theorem 7.1 Any unitary irreducible lowest weight representation of the open string algebra is a tensor product of a unitary irreducible approximately finite representation and a unitary irreducible lowest weight representation in which any element of $\operatorname{sl}(\infty)$ acts as the 0 operator.⿴囗十

Together with the physical interpretation of Theorem 6.2, this result implies that if a lowest weight state has an infinite number of non-zero quantum numbers, it must be a tensor product of a multiple meson state and a state in a representation of the quotient algebra. As remarked in the introduction, the quotient algebra extends and generalizes the Virasoro algebra. Already for the case $\Lambda=1$ the quotient algebra is quite interesting. Specifically, it is an extension of the Virasoro algebra by an infinite Heisenberg algebra 13. Physically speaking, $s l(\infty)$ consists of finite-size-effect operators. Studying the quotient algebra is thus equivalent to studying a physical system which is free of finite-size effects. Hence, we expect the representation theory of the quotient algebra to describe the physics of open matrix chains at the thermodynamic limit.

Let $h$ be the weight function of an arbitrary unitary lowest weight representation $\mathcal{R}$ of the open string algebra, and $\left|v_{h}\right\rangle$ its lowest weight vector (somewhat abusing notation, we do not distinguish between the space and the representation). Our task is to produce two representations $\mathcal{R}_{\text {a.f. }}$ and $\mathcal{R}_{\text {t.i. }}$ such that $\mathcal{R}_{\text {a.f. }}$ is approximately finite, $\mathcal{R}_{\text {t.i. }}$ comes from the quotient

\footnotetext{
${ }^{11}$ This is a corrected version of Theorem 3 in Ref.[16].
} 
algebra (is trivial on $s l(\infty)$ ), and $\mathcal{R}=\mathcal{R}_{\text {t.i. }} \otimes \mathcal{R}_{\text {a.f. }}$. As usual, we do this by proving a succession of lemmas.

Lemma 7.2 In the first two equations below, assume that $\dot{I} \lambda_{1}>\dot{J} \lambda_{2}$, and in the third assume that $\dot{I}>\dot{J}$. Let

$$
\begin{aligned}
\bar{\Xi}_{\lambda_{2}}^{\lambda_{1}} \otimes \tilde{l}_{\dot{J}}^{\dot{I}} & \equiv \bar{\Xi}_{\lambda_{2}}^{\lambda_{1}} \otimes l_{\dot{J}}^{\dot{I}}-\sum_{\lambda_{3}=1}^{\Lambda_{F}} \sum_{\dot{K}} \bar{\Xi}_{\lambda_{2}}^{\lambda_{1}} \otimes f_{\dot{J} \dot{K}}^{\dot{I} \dot{K}} \otimes \Xi_{\lambda_{3}}^{\lambda_{3}}, \\
\tilde{r}_{\dot{J}}^{\dot{I}} \otimes \Xi_{\lambda_{2}}^{\lambda_{1}} & \equiv r_{\dot{J}}^{\dot{I}} \otimes \Xi_{\lambda_{2}}^{\lambda_{1}}-\sum_{\lambda_{3}=1}^{\Lambda_{F}} \sum_{\dot{K}} \bar{\Xi}_{\lambda_{3}}^{\lambda_{3}} \otimes f_{\dot{K} \dot{J}}^{\dot{K} \dot{I}} \otimes \Xi_{\lambda_{2}}^{\lambda_{1}}, \text { and } \\
\tilde{\sigma}_{\dot{J}}^{\dot{I}} & \equiv \sigma_{\dot{J}}^{\dot{I}}-\sum_{\lambda_{1}, \lambda_{2}=1}^{\Lambda_{F}} \sum_{\dot{K}, \dot{L}} \bar{\Xi}_{\lambda_{1}}^{\lambda_{1}} \otimes f_{\dot{K} \dot{K} \dot{L} \dot{L}}^{\dot{K} \dot{L}} \otimes \Xi_{\lambda_{2}}^{\lambda_{2}} .
\end{aligned}
$$

Then $\bar{\Xi}_{\lambda_{2}}^{\lambda_{1}} \otimes \tilde{l}_{\dot{J}}^{\dot{I}}\left|v_{h}\right\rangle, \tilde{r}_{\dot{j}}^{\dot{I}} \otimes \Xi_{\lambda_{2}}^{\lambda_{1}}\left|v_{h}\right\rangle$ and $\tilde{\sigma}_{\dot{J}}^{\dot{I}}\left|v_{h}\right\rangle$ have finite norms.

Proof. We will show that $\tilde{\sigma}_{\dot{j}}^{\dot{I}}\left|v_{h}\right\rangle$ has a finite norm. The rest of the lemma can be proved by a simpler version of the following argument.

For any non-negative integer $p$, consider the operator

$$
\tilde{\sigma}_{\dot{J}}^{\dot{I}}(p)=\sigma_{\dot{J}}^{\dot{I}}-\sum_{\lambda_{1}, \lambda_{2}=1}^{\Lambda_{F}} \sum_{\substack{\dot{K}, \dot{L} \\ \#(\dot{K} \dot{L}) \leq p}} \bar{\Xi}_{\lambda_{1}}^{\lambda_{1}} \otimes f_{\dot{K} \dot{K} \dot{L}}^{\dot{K} \dot{L}} \otimes \Xi_{\lambda_{2}}^{\lambda_{2}} .
$$

Certainly it is well defined because there are only a finite number of summands in Eq. 68). (We can define $\tilde{l}_{\dot{j}}^{\dot{I}}(p)$ and $\tilde{r}_{\dot{j}}^{\dot{I}}(p)$ similarly.) Let

$$
s(\dot{I}, \dot{J}, \dot{K}, \dot{L})=\sum_{\dot{K}^{\prime}, \dot{L}^{\prime}} \delta_{\dot{K}^{\prime} \dot{I} \dot{L}^{\prime}}^{\dot{K} \dot{L}} \delta_{\dot{K}^{\prime} \dot{J} \dot{L}^{\prime}}^{\dot{K} \dot{j} \dot{L}}
$$

Clearly, $s$ is a positive integer. Since

$$
\left[\tilde{\sigma}_{\dot{J}}^{\dot{I}}(p), f_{\dot{K}^{\prime} \dot{I} \dot{L}^{\prime}}^{\dot{K}^{\prime} \dot{j} \dot{L}^{\prime}}\right]=0
$$

for $\#\left(\dot{K}^{\prime} \dot{L}^{\prime}\right) \leq p$

$$
\left\langle v_{h}\left|\tilde{\sigma}_{\dot{I}}^{j}(p) \tilde{\sigma}_{\dot{J}}^{\dot{I}}(p)\right| v_{h}\right\rangle=\left\langle v_{h}\left|\sigma_{\dot{I}}^{\dot{J}} \sigma_{\dot{J}}^{\dot{I}}\right| v_{h}\right\rangle
$$




$$
\begin{aligned}
& -\sum_{\lambda_{1}, \lambda_{2}=1}^{\Lambda_{F}} \sum_{\substack{\dot{K}, \dot{L} \\
\#(\dot{K} \dot{L}) \leq p}} s(\dot{I}, \dot{J}, \dot{K}, \dot{L})\left(h_{I}\left(\lambda_{1} ; \dot{K} \dot{J} \dot{L} ; \lambda_{2}\right)\right. \\
& \left.-h_{I}\left(\lambda_{1} ; \dot{K} \dot{I} \dot{L} ; \lambda_{2}\right)\right),
\end{aligned}
$$

which, in turn, is non-negative owing to unitarity. That $p$ can be arbitrarily large and Lemma [0] together then imply that for any fixed non-empty integer sequences $\dot{I}$ and $J$, only a finite number of

$$
h_{I}\left(\lambda_{1} ; \dot{K} \dot{J} \dot{L} ; \lambda_{2}\right)-h_{I}\left(\lambda_{1} ; \dot{K} \dot{I} \dot{L} ; \lambda_{2}\right),
$$

where $\lambda_{1}$ and $\lambda_{2}$ are arbitrary positive integers not larger than $\Lambda_{F}$, and $\dot{K}$ and $\dot{L}$ are empty or non-empty integer sequences, are non-zero. As a result,

$$
\sum_{\substack{\dot{K}, \dot{L} \\ \#(\dot{K} \dot{L})>q_{0}}} \bar{\Xi}_{\lambda_{1}}^{\lambda_{1}} \otimes f_{\dot{K} \dot{K} \dot{L}}^{\dot{K} \dot{L}} \otimes \Xi_{\lambda_{2}}^{\lambda_{2}}\left|v_{h}\right\rangle=0
$$

for some positive integer $q_{0}$ because its norm vanishes. Thus $\tilde{\sigma}_{\dot{J}}^{\dot{I}}\left|v_{h}\right\rangle$ has a finite norm. Q.E.D.

Define $\mathcal{R}_{f}$ to be the subspace of $\mathcal{R}$ generated by the actions of elements of $g l\left(\Lambda_{F}\right) \otimes F_{\Lambda} \otimes g l\left(\Lambda_{F}\right)$ on the lowest weight vector $\left|v_{h}\right\rangle$. Let for brevity $\tilde{X}$ denote any one of the operators defined in Eqs.(65-67). It now follows easily that $\tilde{X} v$ is well defined for any $v \in \mathcal{R}_{f}$.

Lemma 7.3 For any $v \in \mathcal{R}_{f}$ and any $F \in g l\left(\Lambda_{F}\right) \otimes F_{\Lambda} \otimes g l\left(\Lambda_{F}\right)$,

$$
\tilde{X} F v=F \tilde{X} v .
$$

Proof. Any $F \in g l\left(\Lambda_{F}\right) \otimes F_{\Lambda} \otimes g l\left(\Lambda_{F}\right)$ commutes, for fixed $\dot{I}, \dot{J}$, with everything in $g l\left(\Lambda_{F}\right) \otimes F_{\Lambda} \otimes g l\left(\Lambda_{F}\right)$ of the form $\bar{\Xi}_{\lambda_{1}}^{\lambda_{1}} \otimes f_{\dot{K} \dot{K} \dot{L}}^{\dot{K} \dot{L}} \otimes \Xi_{\lambda_{2}}^{\lambda_{2}}$ except possibly finitely many. The claim now follows by a simple computation as in the proof of the previous lemma. Q.E.D. 
Corollary 7.4 Let $\tilde{X}_{\dot{J}_{p}}^{\dot{I}_{p}}$ stand for either $\bar{\Xi}_{\lambda_{2}}^{\lambda_{1}} \otimes \tilde{l}_{\dot{j}}^{\dot{I}}, \tilde{r}_{\dot{J}}^{\dot{I}} \otimes \Xi_{\lambda_{2}}^{\lambda_{1}}$ or $\tilde{\sigma}_{\dot{J}}^{\dot{I}}$. Then

$$
\prod_{p=1}^{n} \tilde{X}_{\dot{J}_{p}}^{\dot{I}_{p}}\left|v_{h}\right\rangle
$$

has a finite norm for any value of $n$.

Proof. This follows directly from Lemma 7.2 and Lemma 7.3. Q.E.D.

It follows from Lemma 7.2, Lemma 7.3 and Corollary 7.4 that $\tilde{l}, \tilde{r}$ and $\tilde{\sigma}$ are well-defined operators on a lowest weight module.

Lemma 7.5 There exist $\alpha \in \mathbb{R}$ and $N \in \mathbb{N}$ such that for all $\lambda_{1}, \lambda_{2}$ : $h_{I}\left(\lambda_{1} ; I ; \lambda_{2}\right)=\alpha$ provided $\#(I) \geq N$.

Proof. Observe that since $\dot{I}>\dot{J}$, (70) implies more generally for any $\lambda_{1}, \lambda_{2}, \lambda_{3}, \lambda_{4}$ that (the non-negative integer)

$$
\left(h_{I}\left(\lambda_{1} ; \dot{K} \dot{J} \dot{L} ; \lambda_{2}\right)-h_{I}\left(\lambda_{3} ; \dot{K} \dot{I} \dot{L} ; \lambda_{4}\right)\right)
$$

can be non-zero for at most finitely many $\dot{K}, \dot{L}$. As a special case of this, notice that for any $i=1, \ldots, \Lambda_{F}$, only a finite number of

$$
h_{I}\left(\lambda_{1} ; \dot{K} \dot{L} ; \lambda_{2}\right)-h_{I}\left(\lambda_{3} ; \dot{K}\{i\} \dot{L} ; \lambda_{4}\right),
$$

are non-zero. Hence, there exists an $N \in \mathbb{N}$ such that for any $U$ with $\#(U) \geq$ $N$, any $\lambda_{1}, \lambda_{2}, \lambda_{3}, \lambda_{4}, \lambda_{5}, \lambda_{6}$, and any indices $1 \leq i, j \leq \Lambda_{F}, h_{I}\left(\lambda_{1} ; U ; \lambda_{2}\right)=$ $h_{I}\left(\lambda_{3} ; U\{i\} ; \lambda_{4}\right)=h_{I}\left(\lambda_{5} ;\{j\} U ; \lambda_{6}\right)$. But since for any two sequences $U, V$ with $\#(U)=\#(V)=N$ there is a sequence $W$ such that both $U$ and $V$ occur as segments of $W$, it follows that we must have $h_{I}\left(\lambda_{1} ; U ; \lambda_{2}\right)=h_{I}\left(\lambda_{3} ; V ; \lambda_{4}\right)$. Q.E.D.

We can now define the two spaces $\mathcal{R}_{\text {t.i. }}$ (the truly infinite) and $\mathcal{R}_{a . f}$. (the almost finite).

Definition 7.6 Let $\alpha$ be as in Lemma 7.5, set $h_{I}^{\text {a.e. }}=h_{I}-\alpha$, and let $h_{I I}^{a . e .}, h_{I I I}^{a . e}$, and $h_{I V}^{a . e .}$ be defined from $h_{I}^{\text {a.e. }}$ as in Definition $\mathbf{\text { Q. }} \mathcal{R}_{\text {a.f. then }}$ is defined as the lowest weight representation having this lowest weight. Similarly, $\mathcal{R}_{\text {t.i. }}$ is defined to be the lowest weight representation given by the lowest weight $\left(h_{I}^{q}, h_{I I}^{q}, h_{I I I}^{q}, h_{I V}^{q}\right)$ with $h_{I}^{q} \equiv \alpha$, and $h_{W}^{q}=h_{W}-h_{W}^{a . e .}$ for $W=I I, I I I, I V$. 
In the following we shall, among other things, consider elements $f_{\dot{j}}^{\dot{I}}$, with $\dot{I}>\dot{J}$ acting in $\mathcal{R}_{f}$ or in $\mathcal{R}_{a . f}$. We will use the same symbol for these actions since the two spaces are in fact equal as vector spaces. As representations of $g l\left(\Lambda_{F}\right) \otimes F_{\Lambda} \otimes g l\left(\Lambda_{F}\right)$ they differ by a tensor product of a 1-dimensional representation (defined by $\alpha$ ) and this is trivial on said elements. Further-

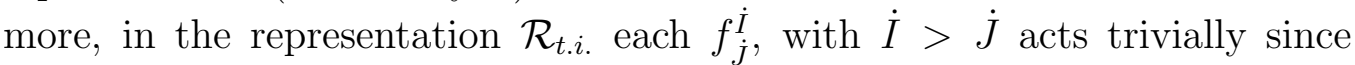
by construction they must annihilate the lowest weight vector while at the same time having commutators with the other generators that again yields elements $f_{\dot{L}}^{\dot{K}}$, with $\dot{K}>\dot{L}$.

Proof of Theorem 11. Let $X_{j_{p}}^{\dot{I}_{p}}$ stand for either $\bar{\Xi}_{\lambda_{2}}^{\lambda_{1}} \otimes l_{\dot{J}}^{\dot{I}}, r_{\dot{J}}^{\dot{I}} \otimes \Xi_{\lambda_{2}}^{\lambda_{1}}$ or $\sigma_{\dot{J}}^{\dot{I}}$ and likewise $\tilde{X}_{\dot{J}_{p}}^{\dot{I}_{p}}$ stand for either $\bar{\Xi}_{\lambda_{2}}^{\lambda_{1}} \otimes \tilde{l}_{\dot{J}}^{\dot{I}}, \tilde{r}_{\dot{j}}^{\dot{I}} \otimes \Xi_{\lambda_{2}}^{\lambda_{1}}$ or $\tilde{\sigma}_{\dot{j}}^{\dot{I}}$. It follows that $\mathcal{R}_{t . i \text {. is }}$ generated by operators of the from $X_{\dot{J}_{p}}^{\dot{I}_{p}}$. Further, it follows from Lemma 7.2 , Lemma 7.3, and Corollary 7.4 that any element of $\mathcal{R}$ can be written as a finite linear combination of elements of the form

$$
\prod_{p=1}^{n} \tilde{X}_{\dot{J}_{p}}^{\dot{I}_{p}} \prod_{p=1}^{n_{I}} \bar{\Xi}_{\rho_{p}^{(I)}}^{\lambda_{p}^{(I)}} \otimes f_{\dot{J}_{p}^{(I)}}^{\dot{I}_{p}^{(I)}} \otimes \Xi_{\zeta_{p}^{(I)}}^{\eta_{p}^{(I)}}\left|v_{h}\right\rangle,
$$

where the two products are arranged in such a way that in each product, the factors follow the lexicographic ordering from Definition 3.2 with $\sigma, r$ and $l$ replaced with $\tilde{\sigma}, \tilde{r}$ and $\tilde{l}$, respectively. Denote the lowest weight vector of $\mathcal{R}_{t . i .}$ by $v_{t . i .}$ and the lowest weight vector of $\mathcal{R}_{a . f}$. by $v_{a . f .}$. Assume they are both unit vectors in their respective spaces. We can then define a surjection from $\mathcal{R}_{\text {t.i. }} \otimes \mathcal{R}_{f}$ to $\mathcal{R}$ by mapping

$$
\left(\prod_{p=1}^{n} X_{\dot{J}_{p}}^{\dot{I}_{p}} v_{t . i .}\right) \otimes\left(\prod_{p=1}^{n_{I}} \bar{\Xi}_{\rho_{p}^{(I)}}^{\lambda_{p}^{(I)}} \otimes f_{\dot{j}_{p}^{(I)}}^{\dot{I}_{(I)}^{(I)}} \otimes \Xi_{\zeta_{p}^{(I)}}^{\eta_{p}^{(I)}} v_{a . f .}\right)
$$

to the one shown in Eq.(73). Because of Lemma 7.3 and the above remarks, this is easily seen to be a map that preserves the respective inner products. By looking at the images of $\mathcal{R}_{t . i .} \otimes v_{a . f .}$ and $v_{t . i .} \otimes \mathcal{R}_{a . f}$. it follows that $\mathcal{R}_{t . i .}$ and $\mathcal{R}_{\text {a.f. }}$ are unitary. The irreducibility is obvious, c.f. Lemma 6.4. Q.E.D.

\section{Acknowledgment}

We thank K. Bering, B. Durhuus, V. John and S. G. Rajeev for discussions. 


\section{References}

[1] T. Banks, W. Fischler, S. Shenker and L. Susskind, Phys. Rev. D55, 5112 (1997) [hep-th/9610043].

[2] E. Witten, Nucl. Phys. B 460, 335 (1996) [hep-th/9510135].

[3] S. Weinberg, The Quantum Theory of Fields, Vols. 1 and 2 (Cambridge University Press, Cambridge, 1996).

[4] T. Banks, TASI Lectures on Matrix Theory [hep-th/9911068];

W. Taylor IV, The M(atrix) Model of M-Theory [hep-th/0002016].

[5] W. Miller, Jr., Symmetry Groups and Their Applications, p.376 (Academic Press, New York and London, 1972)

[6] J. Polchinski, String Theory, Vols. 1 and 2 (Cambridge University Press, Cambridge, 1998).

[7] A. A. Belavin, A. M. Polyakov and A. B. Zamolodchikov, Nucl. Phys. B 241, 333 (1984).

[8] P. Di Francesco, P. Mathieu and D. Sénéchal, Conformal Field Theory (Springer-Verlag, New York, 1997).

[9] C.-W. H. Lee and S. G. Rajeev, Nucl. Phys. B 529, 656 (1998) [hep-th/9712090].

[10] G. 't Hooft, Nucl. Phys. B72, 461 (1974).

[11] Oren Bergman and Charles B. Thorn, Phys. Rev. D 52, 5980 (1995) [hep-th/9506125].

[12] C.-W. H. Lee and S. G. Rajeev, Int. J. Mod. Phys. A 14, 4395 (1999) [hep-th/9906060].

[13] H. P. Jakobsen and C.-W. H. Lee, in preparation.

[14] J. E. Humphreys, Introduction to Lie Algebras and Representation Theory, 2nd ed. (Springer-Verlag, New York, 1978). 
[15] V. G. Kac and A. K. Raina, Bombay Lectures on Highest Weight Representations of Infinite Dimensional Lie Algebras, Advanced Series in Mathematical Physics Vol. 2 (World Scientific, Singapore, 1987).

[16] H. P. Jakobsen and C.-W. H. Lee, Unitary Irreducible Representations of a Lie Algebra for Open Matrix Chains, to appear in the Proceedings of MRST '00.

[17] C. B. Thorn, Phys. Rev. D 20, 1435 (1979). 